\title{
The extended structure of the dwarf irregular galaxies Sextans A and Sextans B
}

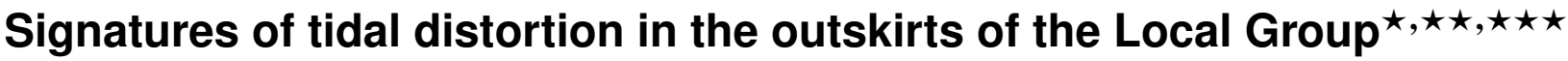

\author{
M. Bellazzini ${ }^{1}$, G. Beccari ${ }^{2}$, F. Fraternali ${ }^{3,5}$, T. A. Oosterloo ${ }^{4,5}$, A. Sollima ${ }^{1}$, V. Testa ${ }^{6}$, S. Galleti $^{1}$, S. Perina $^{7}$, \\ M. Faccini ${ }^{6}$, and F. Cusano ${ }^{1}$ \\ 1 INAF - Osservatorio Astronomico di Bologna, via Ranzani 1, 40127 Bologna, Italy \\ e-mail: michele.bellazzini@oabo.inaf.it \\ 2 European Southern Observatory, Av. Alonso de Córdova, 3107, 19001 Casilla, Santiago, Chile \\ 3 Dipartimento di Astronomia - Università degli Studi di Bologna, via Ranzani 1, 40127 Bologna, Italy \\ ${ }^{4}$ Netherlands Institute for Radio Astronomy, Postbus 2, 7990 AA Dwingeloo, The Netherlands \\ 5 Kapteyn Astronomical Institute, University of Groningen, Postbus 800, 9700 AV Groningen, The Netherlands \\ 6 INAF - Osservatorio Astronomico di Roma, via Frascati 33, 00040 Monteporzio, Italy \\ 7 Institute of Astrophysics, Pontificia Universidad Catolica de Chile, avenida Vicuña Mackenna 4860, 782-0436 Macul, Santiago, \\ Chile
}

Received 17 February 2014 / Accepted 20 March 2014

\section{ABSTRACT}

\begin{abstract}
We present a detailed study of the stellar and H I structure of the dwarf irregular galaxies Sextans A and Sextans B, members of the NGC 3109 association. We use newly obtained deep $(r \simeq 26.5)$ and wide-field $g$ and $r$ photometry to extend the surface brightness (SB) profiles of the two galaxies down to $\mu_{V} \simeq 31.0 \mathrm{mag} / \mathrm{arcsec}^{2}$. We find that both galaxies are significantly more extended than previously traced with surface photometry, out to $\sim 4 \mathrm{kpc}$ from their centres along their major axes. Older stars are found to have more extended distribution than younger populations. We obtain the first estimate of the mean metallicity for the old stars in Sex B, from the colour distribution of the red giant branch, $\langle[\mathrm{Fe} / \mathrm{H}]\rangle=-1.6$. The SB profiles show significant changes of slope and cannot be fitted with a single Sérsic model. Both galaxies have HI discs as massive as their respective stellar components. In both cases the H I discs display solid-body rotation with maximum amplitude of $\sim 50 \mathrm{~km} \mathrm{~s}^{-1}$ (albeit with significant uncertainty due to the poorly constrained inclination), implying a dynamical mass $\sim 10^{9} M_{\odot}$, a mass-to-light ratio $\frac{M}{L_{V}} \sim 25$, and a dark-to-baryonic mass ratio of $\sim 10$. The distribution of the stellar components is more extended than the gaseous disc in both galaxies. We find that the main, approximately round, stellar body of Sex A is surrounded by an elongated low-SB stellar halo that can be interpreted as a tidal tail, similar to that found in another member of the same association (Antlia). We discuss these, as well as other evidence of tidal disturbance, in the framework of a past passage of the NGC 3109 association close to the Milky Way, which has been hypothesised by several authors and is also supported by the recently discovered filamentary configuration of the association itself.
\end{abstract}

Key words. galaxies: dwarf - Local Group - galaxies: structure - galaxies: ISM - galaxies: stellar content

\section{Introduction}

The origin of the variety of morphologies of dwarf galaxies and the evolutionary path leading to the formation of the amorphous and gas-devoid dwarf Spheroidal (dSph) galaxies, are generally recognised as important questions of modern astrophysics, having important consequences for our understanding of galaxy formation and cosmology (Mateo 1998; Tolstoy et al. 2009). The local morphology-density relation (i.e. dSph are found in the sur-

\footnotetext{
* Appendices are available in electronic form at http: //www . aanda.org

$\star \star$ Table of stellar photometry is only available at the CDS via anonymous ftp to cdsarc.u-strasbg. fr (130.79.128.5) or via http: //cdsarc.u-strasbg.fr/viz-bin/qcat?J/A+A/566/A44 $\star \star \star$ Based on data acquired using the Large Binocular Telescope (LBT). The LBT is an international collaboration among institutions in the United States, Italy, and Germany. LBT Corporation partners are The University of Arizona on behalf of the Arizona university system; Istituto Nazionale di Astrofisica, Italy; LBT Beteiligungsgesellschaft, Germany, representing the Max-Planck Society, the Astrophysical Institute Potsdam, and Heidelberg University; The Ohio State University; and The Research Corporation, on behalf of The University of Notre Dame, University of Minnesota and University of Virginia.
}

roundings of giant galaxies while gas-rich dwarf irregulars (dIrr) are farther away, on average) lead to the idea that strong interactions with the large galaxies they are orbiting around transformed primordial actively star-forming dIrrs into present-day quiescent $\mathrm{dSph}$, removing the gas and stopping the star formation (Mateo 1998). Recent state-of-the-art modelling in a cosmological context revealed that tidal interactions and ram-pressure stripping can be quite efficient in transforming gas-rich disc dwarfs (plunging deeply into the halo of their main galaxy) into dSphs (the tidal stirring model, Mayer et al. 2007), but also supernova (SN) winds and the cosmic UV background are found to play a significant rôle in removing gas from dwarfs (Kazantzidis et al. 2011; Sawala et al. 2010, 2012).

Observations of isolated ${ }^{1}$ dwarfs in the Local Group (LG) is expected to provide a crucial insight on the impact of the various

\footnotetext{
1 Here the term isolated means "several hundred kpc distant from a giant galaxy", specifically the Milky Way and M31, since we focus our research on the Local Group and its surrounding, where dwarfs can be resolved into individual stars. In practice, we also selected our targets to lie more than $200 \mathrm{kpc}$ away from other dwarfs. It is obvious that finding a galaxy far away from other LG members at the present epoch does not guarantee that it has evolved in isolation (see, e.g. Teyssier et al. 2012).
} 
factors in shaping the present-day status of dSphs. Isolated dwarfs may represent the test case of evolution without interactions and can provide constraints on the initial conditions at the epoch of formation. These considerations were the driving case for a large Hubble Space Telescope (HST) programme (Gallart 2008) that performs extremely deep observation in small central fields of a few isolated galaxies, searching for the effects of SN feedback and/or cosmic re-ionization, as they may be recorded in the star formation history (SFH) of the systems (see, e.g. Monelli et al. 2010a,b).

We have recently started a research project aimed at exploring a fully complementary aspect, i.e, the large-scale structure of these galaxies, considering both their stellar bodies and their neutral gas components (HI). The lack of tidal limits imposed by nearby masses implies that isolated galaxies may preserve extended stellar haloes at low surface brightness (SB), as has been observed in some cases (e.g. Vansevičius et al. 2004 in Leo A; Sanna et al. 2010 in IC10). These weak but extended structures record crucial information on the formation and evolution of these galaxies, and provide the precious stellar test-particles to study the dynamics of these systems at large distances from their centres, thus probing their dark matter (DM) halos over a wide radial range. The structure and kinematics of the neutral hydrogen provide additional and complementary information. It must be stressed that dwarf galaxies evolved in isolation should have preserved their pristine DM haloes virtually untouched since the beginning of time, hence bearing fundamental information on their original structure and mass distribution.

In a pilot project focused on the extremely isolated dwarf VV124 = UGC4879 (Bellazzini et al. 2011a, hereafter Paper I) we demonstrated that the outer structures of these galaxies can be traced down to extremely low SB levels $\left(\mu_{V} \simeq\right.$ $30 \mathrm{mag} / \operatorname{arcsec}^{2}$ ) with star counts from very deep photometry obtained with state of the art wide-field cameras on $8 \mathrm{~m}$ class telescopes, with modest amounts of observing time, under excellent seeing conditions. In that specific case we found that the stellar body of the galaxy was much more extended than previously believed (from $\sim 500$ to $\gtrsim 1500 \mathrm{pc}$ ), and that the galaxy was highly structured in its outskirts, showing two extended thin wings whose origin is still to be clarified (see Paper I and Kirby et al. 2012, 2013).

In this paper we present the results of the same kind of analysis performed for VV124 in Paper I (and followed up with HST photometry in Bellazzini et al. 2011b), for two additional targets in our survey of nearby isolated dwarfs, the dwarf Irregular (dIrr) galaxies Sextans A (DDO 75) and Sextans B (DDO 70). Both galaxies contain a few $10^{7}$ solar masses of HI (Ott et al. 2012) and are actively forming stars at the rate of $2-8 \times 10^{-3} M_{\odot} \mathrm{yr}^{-1}$ (Weisz et al. 2011). They are members of a very loose group of dwarf galaxies (the NGC 3109 association; see Tully et al. 2006) that is located in the outskirts of the LG, more than $1 \mathrm{Mpc}$ away from the Milky Way (MW) and M31. Triggered by the results shown in Sect. 4 below, we have recently noted that all the known members of the association plus the newly discovered Leo P dwarf (Giovanelli et al. 2013) are strictly clustered around a line in space and display a tight velocity gradient with the distance along this line (Bellazzini et al. 2013). This might indicate that the members of this group may have been subject to a significant tidal interaction in the past.

Sex A and Sex B are separated by more than $250 \mathrm{kpc}$ and they are $\sim 500 \mathrm{kpc}$ and $\sim 700 \mathrm{kpc}$ from the most massive member of the association (NGC 3109), hence they appear as remarkably isolated galaxies at the present epoch. For this reason they were fully eligible as targets for our survey. It is clear that one of the possible outcomes of our analyses is to find out that some of our targets did not evolve in isolation in the past, as now seems to be the case for Sex A, at least. This may open an interesting window on the role of interactions in low-density groups of lowmass galaxies.

The plan of the paper is the following: in Sect. 2 we describe the optical photometry observations and their data reductions and calibrations; in Sect. 3 we provide constraints on the metallicity distribution of old stars, and we briefly consider the spatial distribution of the various stellar species within the two galaxies, an analysis that has never been performed before on such wide scales. In Sect. 4 we present the SB profiles and density maps: even in these cases we are able to trace the stellar structure of the galaxies out to distances from their centres never explored before. In Sect. 5 we re-consider the structure and dynamics of the H I distributions associated with Sex A and Sex B; and, finally, in Sect. 6 we summarise and discuss our results. Moreover, there are two appendices: in Appendix A we present a direct comparison between our own photometry and HST photometry for Sex A and Sex B, while in Appendix B we report on the search for compact star clusters that we performed on our images.

\section{LBT observations and data reduction}

Deep $g$ and $r$ photometry was acquired on the night of February 21, 2012, at LBT, using the Large Binocular Camera (LBC, Giallongo et al. 2008) in binocular mode; $g$ images were acquired with the blue arm and $r$ images with the red arm of the telescope/camera. The optics of each LBC feed a mosaic of four 4608 px $\times 2048$ px CCDs, with a pixel scale of 0.225 arcsec $\mathrm{px}^{-1}$. Each CCD chip covers a field of $17.3^{\prime} \times 7.7^{\prime}$. Chips 1, 2, and 3 flank one another, being adjacent along their long sides; Chip 4 is placed perpendicular to this array, with its long side adjacent to the short sides of the other chips (see Fig. 4 of Giallongo et al. 2008). During our observations the pointing was chosen to place the targets near the centre of Chip 2, with the long side nearly aligned with the major axis of the galaxy (see Fig. 1). In the following, we will use the terms Chip 1 $(2,3,4)$ and field $1(2,3,4)$, abbreviated as $f 1, f 2, f 3$, and $f 4$, interchangeably. Five long $\left(t_{\exp }=300 \mathrm{~s}\right)$ and two short $\left(t_{\exp }=20 \mathrm{~s}\right)$ exposures per filter were acquired during the night; the seeing ranged from $0.70^{\prime \prime}$ to $1.2^{\prime \prime}$. The average seeing and background level were not as good, on average, for the Sex A set of images, resulting in a slightly lower limiting magnitude with respect to the photometry of Sex B ${ }^{2}$. Moreover, Sex B images were obtained at airmass $\simeq 1.13$ while those of Sex A have airmass $\simeq 1.27$. The short exposures were obtained to provide a bridge between the photometry from our long exposures (reaching the saturation level at $r \simeq 18.0$ ) and the secondary calibrators from the SDSS DR9 catalog (Ahn et al. 2012) that can be as faint as $g, r \simeq 23.0$, but have average photometric errors $\sigma_{g}, \sigma_{r} \leq 0.03$ mag only for $g, r \leq 20.0$.

Relative photometry was performed independently on each long exposure image using the point spread function (PSF) fitting code DAOPHOTII/ALLSTAR (Stetson 1987, 1994). Sources with peaks higher than $3 \sigma$ above the background were identified in a stacked image obtained by registering and co-adding all the images considered for the analysis. Then, each of these stars was re-identified and fitted on each image (when possible). Only sources found at least in three $g$ and three

\footnotetext{
2 In particular for the deep $r$ images, with a mean seeing of $1.08^{\prime \prime}$ and $0.86^{\prime \prime}$ for Sex A and Sex B, respectively.
} 

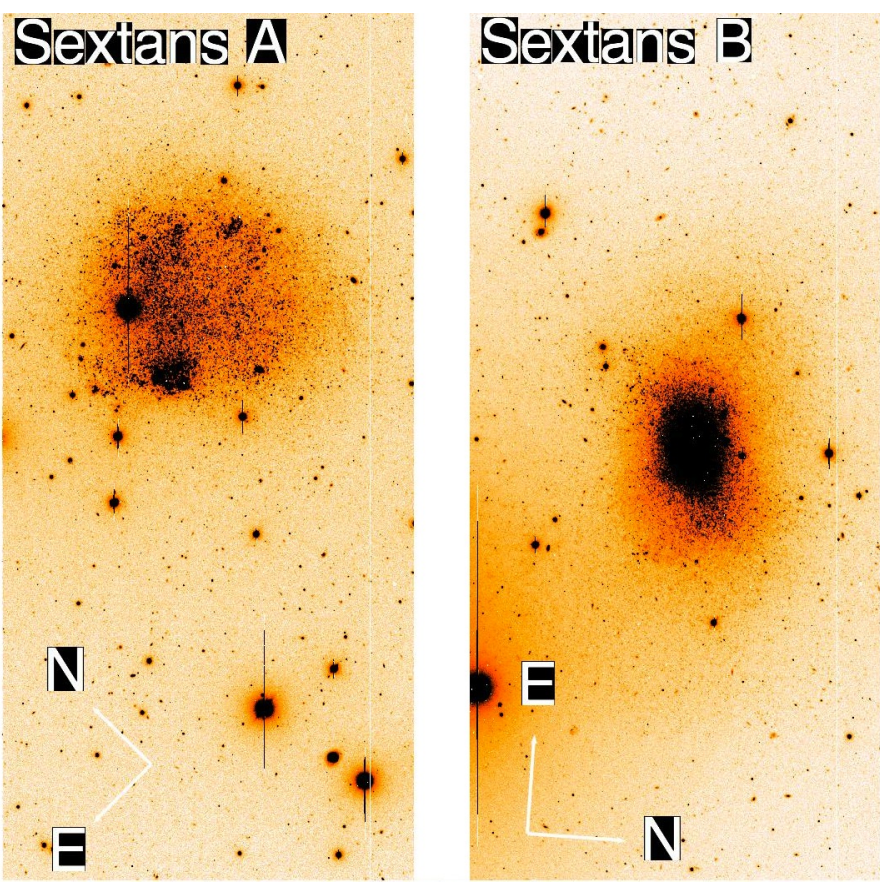

Fig. 1. Deep $g$-band images of a large portion of Chip 2 for Sex A (left panel) and Sex B (right panel). The sampled FoV is $7.7^{\prime} \times 16.2^{\prime}$.

$r$ images were retained in the final catalog. The average and the standard error of the mean of the independent measures obtained from the different images were adopted as the final values of the instrumental magnitude and of the uncertainty on the relative photometry. To clean the catalog from spurious sources, we also adopted cuts in the image quality parameters CHI and SHARP, provided by DAOPHOTII. After accurate inspection of the distribution of measured sources in the planes mag vs. CHI and mag vs. SHARP, we decided to retain only sources having $\mathrm{CHI} \leq 2.0$ and SHARP within magnitude-dependent contours traced to include the bulk of stellar objects. Considering all four fields together, this selection removed 12745 (15 864) sources from the Sex A (Sex B) original dataset, leaving a final catalog of 52598 (68 369) retained sources. Aperture photometry (corrected to infinite apertures) was independently obtained for short exposures images using Sextractor (Bertin \& Arnouts 1996).

\subsection{Astrometry and photometric calibration}

\subsubsection{Sextans B}

The instrumental positions were transformed (independently for each chip) into J2000 celestial coordinates using the stars in common with the SDSS DR9 catalog (Ahn et al. 2012). The adopted astrometric solutions, in the form of third-degree polynomials, were obtained with CataXcorr ${ }^{3}$ using more than 100 stars per field; the root mean square (rms) scatter of the solutions was $\leq 0.1^{\prime \prime}$ in both RA and Dec.

The instrumental magnitudes $\left(g_{i}, r_{i}\right)$ were also transformed into the SDSS ugriz absolute photometric system using stars in common with SDSS DR9, with a first-order polynomial as in Paper I. In particular, photometry from short exposures was

\footnotetext{
3 CataXcorr is a code aimed at cross-correlating catalogs and finding astrometric solutions, developed by P. Montegriffo at INAF Osservatorio Astronomico di Bologna, and successfully used by our group during the past 10 years.
}

calibrated first and then the solution was transferred to the main catalog obtained from deep images. The rms scatter about the photometric solutions is $\simeq 0.04 \mathrm{mag}$, implying an error on the zero point of $\simeq 0.01 \mathrm{mag}$. In the following analysis we will use Eqs. (3)-(6) of Paper I to transform $g, r$ magnitudes into $V, I$, when needed.

\subsubsection{Sextans $A$}

Sex A is not included in the SDSS footprint, hence photometric secondary standards are lacking in the field. Since the observations were acquired within a few hours of those of Sex B we adopted the same photometric solution, corrected for the difference in airmass between the two sets of observations. Clearly, the absolute photometric calibration of the Sex A data is more uncertain than that of Sex B. Still, a direct comparison with the HST photometry publicly provided by the ANGST team (Dalcanton et al. 2009) ${ }^{4}$ shows that the zero points of the two datasets are in satisfactory agreement (within $\sim 0.03$ mag for Sex B and within $\sim 0.10 \mathrm{mag}$ for Sex A). We note that a very accurate photometric calibration for this kind of data is both difficult to achieve (see Paper I, for discussion) and not crucial for the scientific goals of the present survey. The astrometric solution (analogous to that described above for Sex B) was derived from GSC2.2 standard stars, using more than 100 stars per field; the rms scatter of the solutions was $\leq 0.3^{\prime \prime}$ in both RA and Dec. A final cross-check with the catalog by Massey et al. (2007) revealed a rms scatter $\leq 0.15^{\prime \prime}$ in both RA and Dec, for the stars in common with this dataset.

\subsection{Reddening and distance}

We interpolated the Schlegel et al. (1998) reddening maps, as recalibrated by Schlafly \& Finkbeiner (2011), to obtain an estimate of $E(B-V)$ and its variation over the considered fields of view (FoVs). We adopted a regular grid with knots spaced at 3.0', and found that the mean reddening values are slightly lower than those usually reported in the literature (see, e.g. Dalcanton et al. 2009; McConnachie 2012, hereafter M12), due to the Schlafy et al. re-calibration, and the reddening variations are negligible (see Tables 1 and 2). In the following, as in Paper I, we adopt the reddening laws $A_{g}=3.64 E(B-V)$ and $A_{r}=2.71 E(B-$ $V$ ), derived by Girardi et al. (2004) for cool metal-poor giants. We note that the choice of the reddening law is not particularly relevant in these cases, given the very low extinction regime of the considered fields.

To obtain distances fully consistent with the scale adopted in Paper I we used the accurate $V, I$ HST photometry from ANGST to correct the ANGST CMD for the newly derived values of the reddening and re-derive the magnitude and mean colours of the RGB tip as done in Paper I. We obtain $I_{0, \text { tip }}=21.72 \pm 0.06$ and $(V-I)_{0, \text { tip }}=1.39$ for Sex A, and $I_{0, \text { tip }}=21.75 \pm 0.06$ and $(V-I)_{0, \text { tip }}=1.41$ for Sex B, in excellent agreement with Dalcanton et al. (2009). Using the following equation for the absolute magnitude of the RGB tip,

$M_{I}^{\mathrm{TRGB}}=0.080(V-I)_{0}^{2}-0.194(V-I)_{0}-3.939$,

from Bellazzini $(2008)^{5}$, we obtain $M_{I}^{\mathrm{TRGB}}=-4.05 \pm 0.10$, for both galaxies. Finally, we obtain $(m-M)_{0}=25.77 \pm 0.12$ for

\footnotetext{
4 http://www . nearbygalaxies.org/

5 Derived from the original calibrations as a function of $[\mathrm{Fe} / \mathrm{H}]$ and $[\mathrm{M} / \mathrm{H}]$ presented in Bellazzini et al. $(2001,2004)$.
} 
Table 1. Observed and derived parameters of Sextans A.

\begin{tabular}{|c|c|c|}
\hline Parameter & Value & Notes \\
\hline$\alpha_{0}$ & $10: 11: 00.1$ & $\mathrm{~J} 2000^{a}$ \\
\hline$\delta_{0}$ & $-04: 41: 42.6$ & $\mathrm{~J} 2000^{a}$ \\
\hline$l_{0}$ & $246.15^{\circ}$ & Gal. long. \\
\hline$b_{0}$ & $39.87^{\circ}$ & Gal. lat. \\
\hline SGL & $109.00^{\circ}$ & Supergal. long. \\
\hline SGB & $-40.66^{\circ}$ & Supergal. lat. \\
\hline$E(B-V)$ & $0.035 \pm 0.002$ & Average $\pm \sigma^{b}$ \\
\hline$(m-M)_{0}$ & $25.77 \pm 0.12$ & \\
\hline$D$ & $1.42 \pm 0.08 \mathrm{Mpc}$ & \\
\hline $1 \operatorname{arcsec}$ & $6.9 \mathrm{pc}$ & conv. factor at $\mathrm{D}=1.42 \mathrm{Mpc}$ \\
\hline$V_{\mathrm{h}}$ & $324 \pm 1 \mathrm{~km} \mathrm{~s}^{-2}$ & heliocentric velocity ${ }^{c}$ \\
\hline$V_{\mathrm{g}}$ & $163 \mathrm{~km} \mathrm{~s}^{-1}$ & galactocentric velocity ${ }^{c}$ \\
\hline$\langle[\mathrm{Fe} / \mathrm{H}]\rangle$ & -1.45 & from RGB colour ${ }^{d}$ \\
\hline$\epsilon$ & 0.0 & adopted \\
\hline PA & $0^{\circ}$ & adopted \\
\hline$\mu_{V}(0)$ & $23.9 \pm 0.1 \mathrm{mag} / \operatorname{arcsec}^{2}$ & central SB ${ }^{e}$ \\
\hline$h$ & $2.5^{\prime}$ & Sérsic scale radius \\
\hline$r_{\mathrm{h}}$ & $1.8^{\prime}$ & Observed $^{f}$ \\
\hline$V_{\text {tot }}$ & $11.7 \pm 0.2$ & Observed $^{f}$ \\
\hline$M_{V}$ & $-14.2 \pm 0.3$ & \\
\hline$L_{V}$ & $4.1_{-1.0}^{+1.3} \times 10^{7} L_{V, \odot}$ & total V luminosity \\
\hline$M_{\mathrm{HI}}$ & $6.2 \times 10^{7} M_{\odot}$ & gaseous mass $^{g}$ \\
\hline$M_{\mathrm{dyn}}$ & $\sim 1 \times 10^{9} M_{\odot}$ & total mass ${ }^{h}$ \\
\hline
\end{tabular}

Notes. ${ }^{(a)}$ Estimated by eye from our images. ${ }^{(b)}$ Averaged over the whole FoV from the reddening maps by Schlegel et al. (1998), as recalibrated by Schlafly \& Finkbeiner (2011). (c) From McConnachie (2012). ${ }^{(d)}$ From synthetic CMD fit on HST photometry by Dolphin et al. (2003). ${ }^{(e)}$ Average of $\mu_{V}$ for $R_{\epsilon}<1.8^{\prime}$. Not corrected for extinction. The $\mu_{V}(0)$ values extrapolated from fits with not fully adequate models (e.g. exponential models) can differ significantly from this $o b$ served value. ${ }^{(f)}$ From the numerical integration of the SB profile in $r_{\epsilon}$. ${ }^{(g)}$ From Ott et al. (2012). ${ }^{(h)}$ See Sect. 5.

Sex A, and $(m-M)_{0}=25.80 \pm 0.12$ for Sex B, corresponding to $D=1.42 \pm 0.08 \mathrm{Mpc}$ and $D=1.44 \pm 0.08 \mathrm{Mpc}$, respectively, where the reported errors include all the statistic and systematic sources of error. The derived distance moduli are slightly larger than those by Dalcanton et al. (2009), because of the small differences in the adopted reddening and $M_{I}^{\mathrm{TRGB}}$, they are still fully compatible within the uncertainties.

\subsection{The colour-magnitude diagrams}

In Fig. 2 we present our final chip-by-chip colour-magnitude diagrams (CMD) for Sex A and Sex B. The quality of the CMDs is similar to Paper I; also in the present case we barely reach the red clump level in the outermost, less crowded regions. As known from previous studies (in particular Dohm-Palmer et al. 1997a; Dalcanton et al. 2009) the CMDs of the two galaxies are similar: they are dominated by a strong and wide red giant branch (RGB), ranging from $(r, g-r) \sim(27.0,0.4)$ to $\sim(22.5,1)$. Above the RGB Tip sparse population of bright asymptotic giant branch (AGB) stars, revealing the presence of an intermediate-age population, can be seen, more abundant in Sex B than in Sex A. On the other hand, the young main sequence (MS) plume at the blue edge of the CMDs is much more prominent in Sex A, where the separation between H-burning MS and He-burning blue loop (BL) stars is also evident for $r \leq 23.0$, MS stars populating the bluer of the two parallel features. A plume of red super giants (RSGs) is also evident around $g-r \sim 1.0$, from $r \sim 22.5$ to
Table 2. Observed and derived parameters of Sextans B.

\begin{tabular}{|c|c|c|}
\hline Parameter & Value & Notes \\
\hline$\alpha_{0}$ & 10:00:00.05 & $\mathrm{J} 2000^{a}$ \\
\hline$\delta_{0}$ & $+05: 19: 56.4$ & $\mathrm{~J} 2000^{a}$ \\
\hline$l_{0}$ & $233.20^{\circ}$ & Gal. long. \\
\hline$b_{0}$ & $43.78^{\circ}$ & Gal. lat. \\
\hline SGL & $95.46^{\circ}$ & Supergal. long. \\
\hline SGB & $-39.62^{\circ}$ & Supergal. lat. \\
\hline$E(B-V)$ & $0.024 \pm 0.003$ & Average $\pm \sigma^{b}$ \\
\hline$(m-M)_{0}$ & $25.80 \pm 0.12$ & \\
\hline$D$ & $1.44 \pm 0.08 \mathrm{Mpc}$ & \\
\hline $1 \operatorname{arcsec}$ & $7.0 \mathrm{pc}$ & conv. factor at $D=1.44 \mathrm{Mpc}$ \\
\hline$V_{\mathrm{h}}$ & $304 \pm 1 \mathrm{~km} \mathrm{~s}^{-1}$ & heliocentric velocity ${ }^{c}$ \\
\hline$V_{g}$ & $171 \mathrm{~km} \mathrm{~s}^{-1}$ & galactocentric velocity $^{c}$ \\
\hline$\langle[\mathrm{Fe} / \mathrm{H}]\rangle$ & -1.6 & from RGB colour \\
\hline$\epsilon$ & 0.31 & adopted ellipticity $^{c}$ \\
\hline PA & $95^{\circ} \pm 15^{\circ}$ & from $R \leq 150^{\prime \prime}$ \\
\hline$\mu_{V}(0)$ & $23.1 \pm 0.1 \mathrm{mag} / \operatorname{arcsec}^{2}$ & central $\mathrm{SB}^{d}$ \\
\hline$h$ & $2.0^{\prime}$ & Sérsic scale radius ${ }^{e}$ \\
\hline$r_{\mathrm{h}}$ & $1.9^{\prime}$ & Observed $^{f}$ \\
\hline$V_{\text {tot }}$ & $11.7 \pm 0.1$ & Observed $^{f}$ \\
\hline$M_{V}$ & $-14.2 \pm 0.2$ & \\
\hline$L_{V}$ & $4.1_{-0.7}^{+0.8} \times 10^{7} L_{V, \odot}$ & total $V$ luminosity \\
\hline$M_{\mathrm{HI}}$ & $4.1 \times 10^{7} M_{\odot}$ & gaseous mass $^{g}$ \\
\hline$M_{\mathrm{dyn}}$ & $\sim 1 \times 10^{9} M_{\odot}$ & total mass ${ }^{h}$ \\
\hline
\end{tabular}

Notes. ${ }^{(a)}$ From Cotton et al. (1999). ${ }^{(b)}$ Averaged over the whole FoV from the reddening maps by Schlegel et al. (1998), as re-calibrated by Schlafly \& Finkbeiner (2011). (c) From McConnachie (2012). (d) Average of $\mu_{V}$ for $r_{\epsilon} \leq 0.5^{\prime}$. Not corrected for extinction. The $\mu_{V}(0)$ values extrapolated from fits with not fully adequate models (e.g. exponential models) can differ significantly from this observed value. (e) From the best-fit model for the entire profile (continuous line in Fig. 7). ${ }^{(f)}$ From the numerical integration of the SB profile in $r_{\epsilon}$. (g) From Ott et al. (2012). ${ }^{(h)}$ See Sect. 5.

$r \sim 20.0$ (see Dohm-Palmer et al. 1997b, for a detailed discussion and identification of all these features in the CMD of Sex A; for a comparison with HST CMDs see Appendix A).

At odds with the case presented in Paper I, the stellar body of both galaxies exceeds $f 2$ : the characteristic RGB is also clearly present in $f 1$ and $f 3$, while $f 4$ can be taken as a reasonable representation of the fore/background contamination affecting our CMDs. Following the detailed discussion presented in Paper I it is easy to identify (especially in both the $f 4$ CMDs) the thin vertical plume spanning the whole diagram around $g-r \simeq 1.5$, due to local $\mathrm{M}$ dwarfs, and the sparse wide band around $g-r \simeq 0.6$, bending to the red for $r \gtrsim 22.0$, due to foreground MS stars in the Galactic halo, while the broad blob of sources with $0.0 \lesssim$ $g-r \lesssim 1.0$ and $r \gtrsim 23.5$ is largely dominated by distant unresolved galaxies (see Paper I for a detailed discussion).

\subsection{Artificial star experiments}

The completeness of the stellar catalogs has been estimated by means of extensive artificial star experiments, limited to Chip 2, since in both the cases this field samples the whole range of crowding encountered in the total LBC FoV. Following the same procedure adopted in Paper I, a total of $\sim 100000$ artificial stars per galaxy have been added to the images and the entire data reduction process has been repeated as in the real case, also adopting the same selection criteria described above. The PSF adopted as the best-fit model for photometry was also assumed 

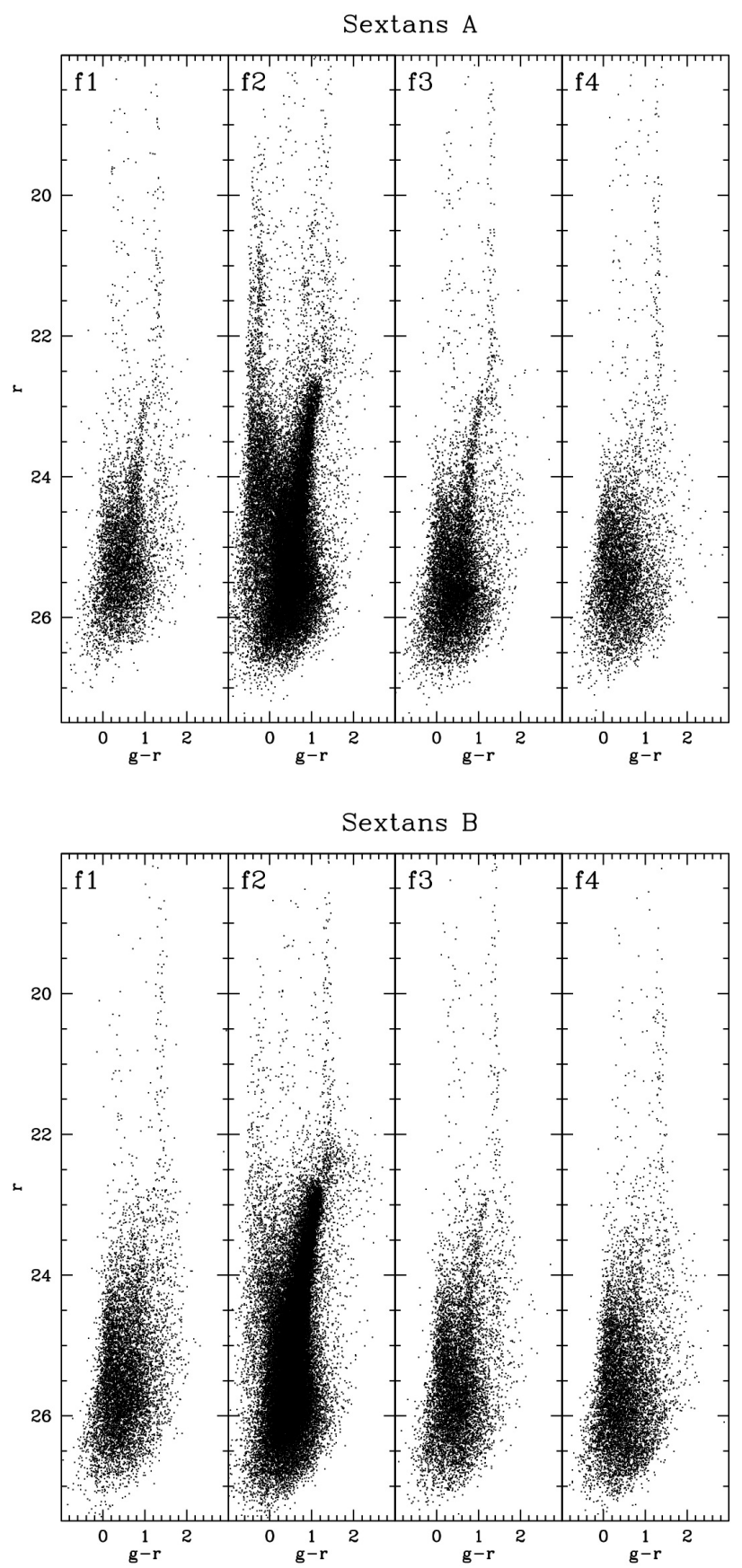

Fig. 2. Chip-by-chip colour-magnitude diagrams of Sex A (upper panels) and Sex B (lower panels).

as the model for the artificial stars. Artificial stars were distributed uniformly in position, over the entire extent of $f 2$, and in colour, over the range $-0.6 \leq g-r \leq 2.0$. They were distributed in magnitude according to a luminosity function similar to the observed one, but monotonically increasing even beyond the limit of the photometry, down to $r \simeq 27.5$ (see Bellazzini et al. 2002a, for details and discussion).

In Fig. 3, we show the completeness fraction $\left(C_{f}\right)$ as a function of $r$ magnitude for different colour ranges and for different (elliptical) radial ranges (see Sect. 2.5, for a definition of $r_{\epsilon}$ ), and the difference between input and output magnitudes for all the artificial stars recovered. The significantly higher degree of crowding in the inner regions of Sex B, compared to Sex A,
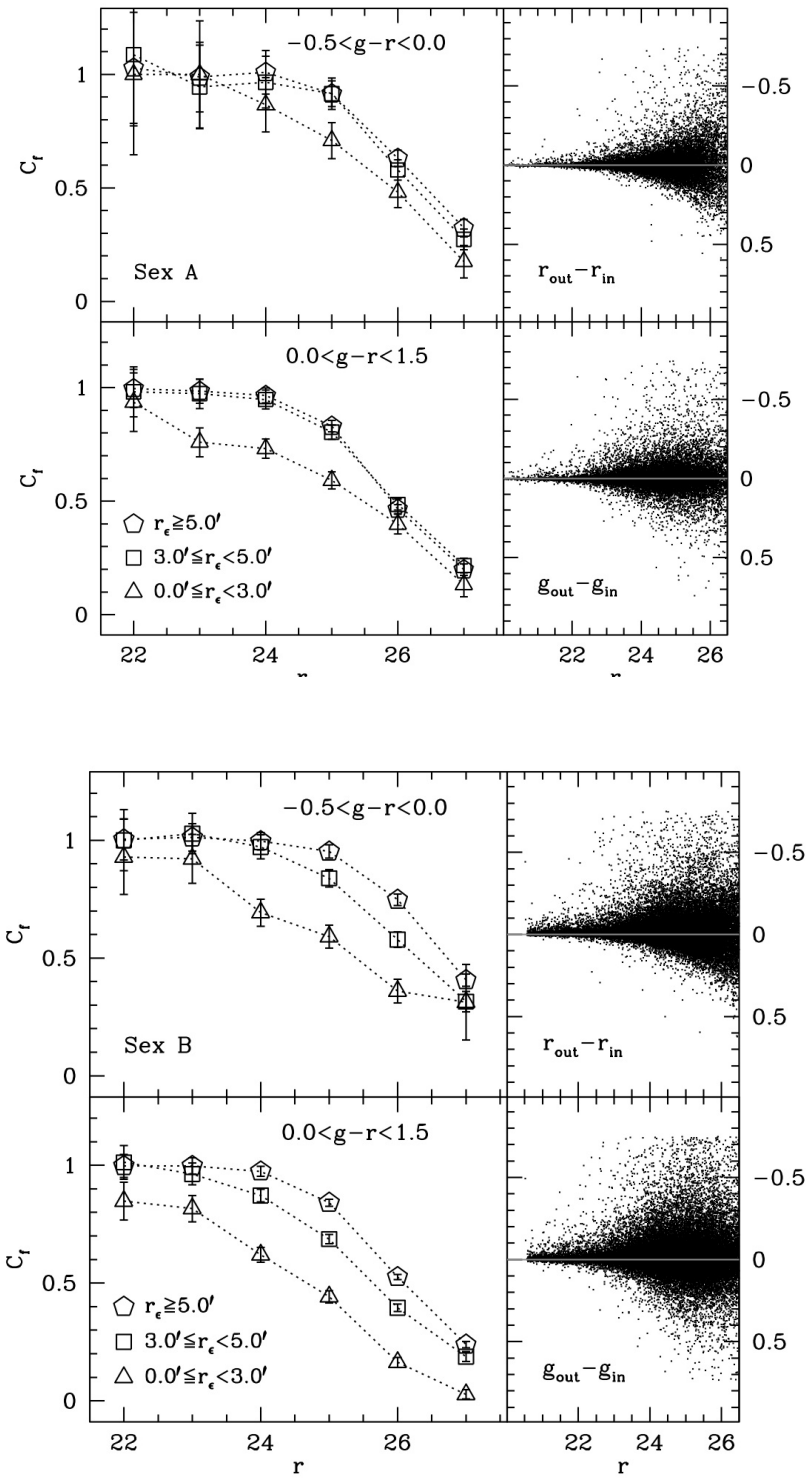

Fig. 3. Completeness fraction as a function of $r$ magnitude for different colour ranges (upper and lower panels) and for different (elliptical) radial ranges (different symbols), for Sex A (upper panel) and Sex B (lower panel).

is at the origin of the difference in the radial behaviour of the completeness as well as in the relative photometric accuracy. As quantitative reference, in Table 3 we provide the standard deviations of the input-output magnitude differences from artificial stars in various magnitude bins. These are average values over the whole 22 field, the actual accuracy depending on the distance from the central regions of the galaxies.

\subsection{Surface photometry and coordinate system}

As a basis for the construction of the final composite profiles (surface photometry + star counts; see Sect. 4) we adopted the surface photometry by Hunter \& Elmegreen (2006, hereafter HE06), kindly provided by D. Hunter. The surface photometry of HE06 extends to relatively large radii $\left(198^{\prime \prime}\right.$ and $280^{\prime \prime}$ for Sex A and Sex B, respectively) and it is carefully corrected for the contribution of several bright foreground stars superimposed onto the main body of the two galaxies. For a sanity check 
Table 3. Accuracy of the relative photometry from artificial star experiments ${ }^{a}$.

\begin{tabular}{lcccc}
\hline \hline & Sex A & \multicolumn{3}{c}{ Sex B } \\
\hline mag range & $\sigma_{g}$ & $\sigma_{r}$ & $\sigma_{g}$ & $\sigma_{r}$ \\
$r<22.0$ & 0.02 & 0.01 & 0.03 & 0.02 \\
$22.0 \leq r<23.0$ & 0.05 & 0.03 & 0.06 & 0.05 \\
$23.0 \leq r<24.0$ & 0.06 & 0.04 & 0.08 & 0.06 \\
$24.0 \leq r<25.0$ & 0.09 & 0.07 & 0.11 & 0.08 \\
$25.0 \leq r<26.0$ & 0.12 & 0.12 & 0.15 & 0.12 \\
\hline
\end{tabular}

Notes. ${ }^{(a)}$ Averaged over the whole $\mathrm{f} 2$ field of each set of observations.

we also performed surface photometry in the innermost 144" of the best $g$ and $r$ images using XVISTA $^{6}$ (see Lauer 1985; Federici et al. 2007; and Paper I, for details on the code and on the adopted procedure). In the overlapping regions the shapes of the profiles obtained with XVISTA from our images and those by HE06 are in excellent agreement.

Inspecting the ellipticity $(\epsilon)$ and position angle (PA) profiles we decided (a) to consider Sex A as round, as a basic approximation for the purpose of deriving the SB profile, given its low ellipticity and, consequently, poorly determined PA $(\epsilon=0.17$ and PA $=0^{\circ}$, according to M12; $\epsilon=0.15$ and PA $=41.8^{\circ}$, according to HE06); (b) to adopt for Sex B PA $=95^{\circ} \pm 15^{\circ}$, from the average of the $\mathrm{g}$ and $\mathrm{r}$ profiles within $R \leq 144^{\prime \prime}$ obtained with XVISTA, in reasonable agreement with the estimates reported by $\mathrm{M} 12\left(\mathrm{PA}=110^{\circ}\right)$ and $\mathrm{HE} 06\left(\mathrm{PA}=87.6^{\circ}\right)$; and $(\mathrm{c})$ again for Sex B, to adopt $\epsilon=0.31$ from M12, as we found it more appropriate over the whole body of the galaxy than the typical value we obtained with XVISTA $(\epsilon \simeq 0.4)$ and estimated by HE06 ( $\epsilon \simeq 0.41)$. All these choices, as well as the position of the centres of the galaxies, have been verified to be adequate for the stellar components of the galaxies (and preferable to values found in the literature) by detailed visual inspection of our images, and comparison with superposed ellipses with the proper parameters. For the shape parameters of the H I components see Sect. 5. It must be noted that these galaxies have, by definition, irregular morphologies and the adoption of a single value of $\epsilon$ and PA over the whole extension of the galaxies is just a convenient approximation that allows us to parametrise the overall morphology with a simple model (see, e.g. HE06 for discussion).

Assuming the coordinates for the centre listed in Tables 1 and 2, we convert to projected cartesian coordinates $X, Y$ (in arcmin) and adopt the elliptical radius $r_{\epsilon}$, equivalent to the majoraxis radius, as the radial coordinate of reference (see Paper I for references and definitions).

\section{Population gradients}

In Fig. 4 we compare the CMDs of Sex A and Sex B in different radial ranges with the ridge lines of two metal-poor globular clusters, M92 $([\mathrm{Fe} / \mathrm{H}]=-2.31)$ and M3 $([\mathrm{Fe} / \mathrm{H}]=-1.50$; see Harris 1996; Carretta et al. 2009). The ridge lines are from the set by Clem et al. (2008), converted from $g^{\prime}, r^{\prime}$ to $g, r$ according to Tucker et al. (2006), as in Paper I. The assumed reddening and distance moduli of the clusters are from the 2010 version of the Harris (1996) catalog.

The radial thresholds have been chosen (a) to clearly separate the innermost region, which contains most of the young stars and being maximally affected by the crowding, from the rest of

\footnotetext{
6 http://astronomy.nmsu.edu/holtz/xvista/index.html
}
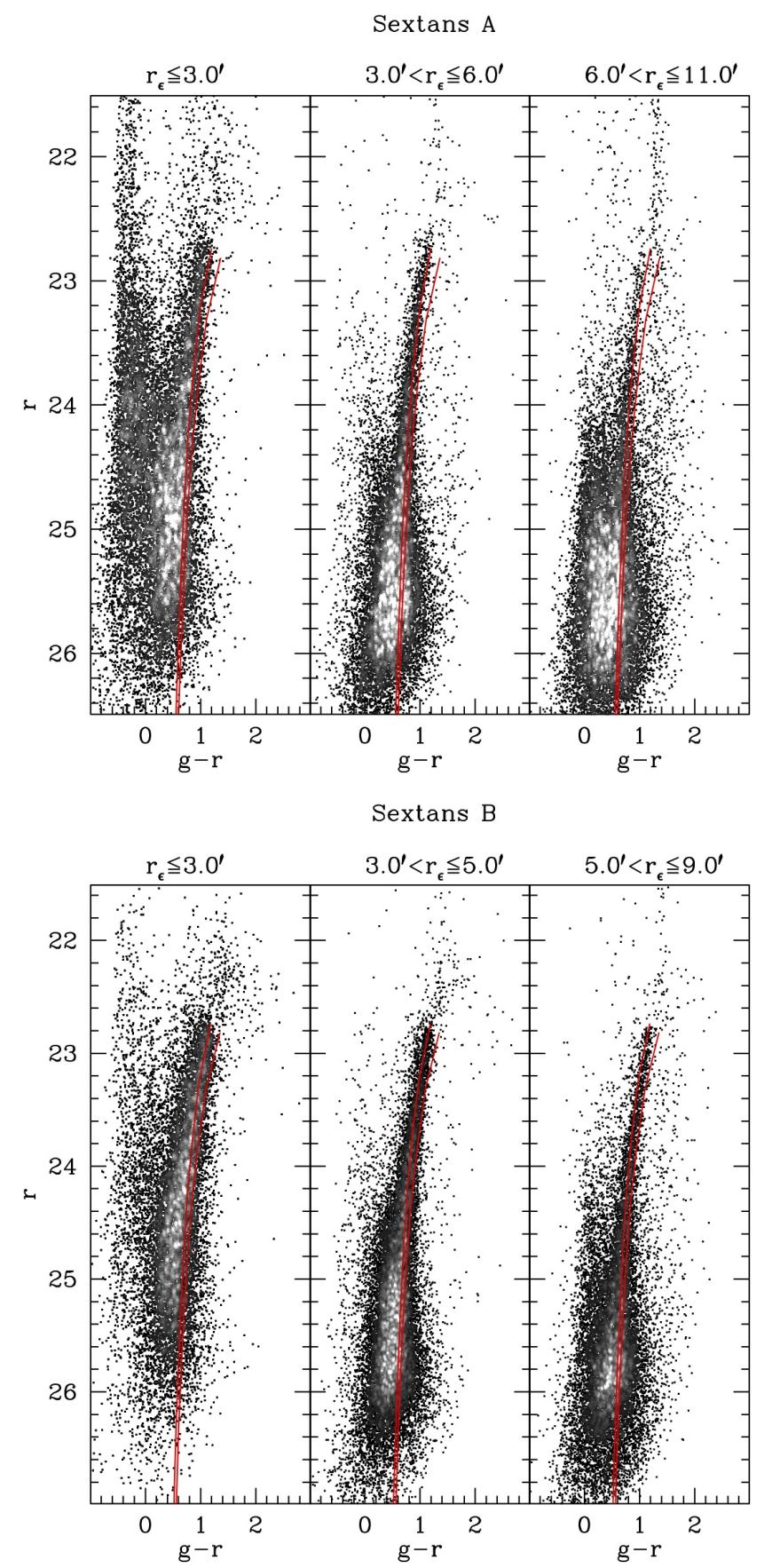

Fig. 4. CMDs for Sex A (upper panels) and Sex B (lower panels) in different radial ranges. The red giant branches are compared with the ridge lines of the globular clusters M92 $([\mathrm{Fe} / \mathrm{H}]=-2.31)$ and $\mathrm{M} 3$ $([\mathrm{Fe} / \mathrm{H}]=-1.50)$, from left to right. In all the panels stars are plotted as black points in regions of the CMD with few stars and as grey squares otherwise, with the scale of grey proportional to the local density in the CMD. Lighter shades of grey correspond to higher density.

the galaxy, where the quality of the photometry and the completeness varies only very mildly with radius; and (b) to look for differences in the stellar populations in correspondence to breaks in the SB profiles (see Sect. 4). The overall extent of the SB profiles was the motivation for the choice of the outermost $r_{\epsilon}$ limit considered in Fig. 4.

The basic conclusion that can be drawn from Fig. 4 is that, in both galaxies, there is no obvious difference in the colour distribution of RGB stars in the different radial ranges considered. 
The old to intermediate-age population traced by these stars have colours typical of metal-poor RGB stars (in general $[\mathrm{Fe} / \mathrm{H}] \lesssim$ $-1.5)$ at any radius in both galaxies. The RGB appears slightly bluer in Sex A than in Sex B, suggesting a lower mean metallicity and/or a lower mean age in the first galaxy. It must be noted that the absolute calibration of our Sex A photometry is more uncertain, and this may play a role in this context. Still, the RGB of Sex A lies on the blue side of the RGB of Sex B even when the HST CMDs are compared, strongly suggesting that the difference observed in Fig. 4 is real. Weisz et al. (2011) found that Sex B formed $>50 \%$ of its stars more than 12.5 Gyr ago, while Sex A reached the same fraction of star formation only $5 \mathrm{Gyr}$ ago, pointing to a younger average age as the main driver of the difference in the RGB colour between the two galaxies.

Concerning the metallicity, M12 reports $\langle[\mathrm{Fe} / \mathrm{H}]\rangle \sim-1.85$ for Sex A, from the mean $V-I$ colour of the RGB determined from the shallow photometry by Sakai et al. (1996), while from the fit of their HST CMD Dolphin et al. (2003) estimated $\langle[\mathrm{M} / \mathrm{H}]\rangle=-1.45$, with a spread of \pm 0.20 dex.

On the other hand, no abundance estimate for the old to intermediate-age of Sex B is available. Given the large fraction of very old stars that should populate the RGB of Sex B (according to the SFH derived by Weisz et al. 2011), the colour of this sequence should provide a relatively reliable indication of the metallicity, when compared with old-age templates. Figure 4 suggests that the vast majority of RGB stars in Sex B should have $-2.3 \lesssim[\mathrm{Fe} / \mathrm{H}] \lesssim-1.5$. We obtain individual estimates for the RGB stars of Sex B in the ANGST HST sample lying within one magnitude from the tip, by interpolating on a grid of globular cluster ridge lines as done in Bellazzini et al. (2002b). From this set of individual metallicities we obtain a mean metallicity $\langle[\mathrm{Fe} / \mathrm{H}]\rangle=-1.6$, with a standard deviation of $0.35 \mathrm{dex}$, in the Carretta \& Gratton (1997) metallicity scale.

Kniazev et al. (2005) and Magrini et al. (2005) derived abundance estimates for the young populations in both galaxies from spectroscopic analysis of a few HII regions, finding typical metallicity values around 0.05-0.1 solar. Kaufer et al. (2004) derived spectroscopic metallicity for three RSG in Sex A, finding a mean $[\mathrm{Fe} / \mathrm{H}] \simeq-1.0$, in reasonable agreement with the above results, and $[\alpha / \mathrm{Fe}] \simeq-0.1$. The comparison between the typical metallicity of the oldest stars $(\mathrm{RGB},[\mathrm{Fe} / \mathrm{H}] \lesssim-1.5)$ and the youngest stars (OB stars in $\mathrm{HII}$ regions, $\mathrm{Fe} / \mathrm{H}] \sim-1.0)$ implies a moderate degree of chemical enrichment over the lifetime of the galaxies.

In the following of this section we provide a first broad look at the relative spatial distribution of stars tracing different epochs of star formation. A more detailed analysis, also including the clustering properties of the young populations (that are known to be fairly complex, at least in Sex A, see Dohm-Palmer et al. 1997b), also in relation with the distribution of HI, is deferred to a future contribution. In Fig. 5 we show the boxes we adopt (in both galaxies) to select (a) MS and BL stars, tracing the youngest stellar populations (age $\$ 300 \mathrm{Myr}$ ); (b) bright AGB stars, tracing itermediate-age populations (up to age $\sim 5 \mathrm{Gyr}$ ); and (c) RGB stars, tracing intermediate/old populations (between $\sim 2 \mathrm{Gyr}$ and $\sim 13 \mathrm{Gyr}$ ). In the right panel of the figure we superimpose the same boxes on a CMD sampling the fore/background population, to provide a direct idea of the effect of contamination on the radial distribution of the various tracers. The MS+BL box is virtually unaffected by contamination. The effect should also be negligible for the RGB box, for $r<24.0$; on the other hand, the sparse population of AGB stars can be significantly contaminated by foreground $\mathrm{M}$ dwarfs even at the centre of the considered galaxies. Therefore, it must be taken into account

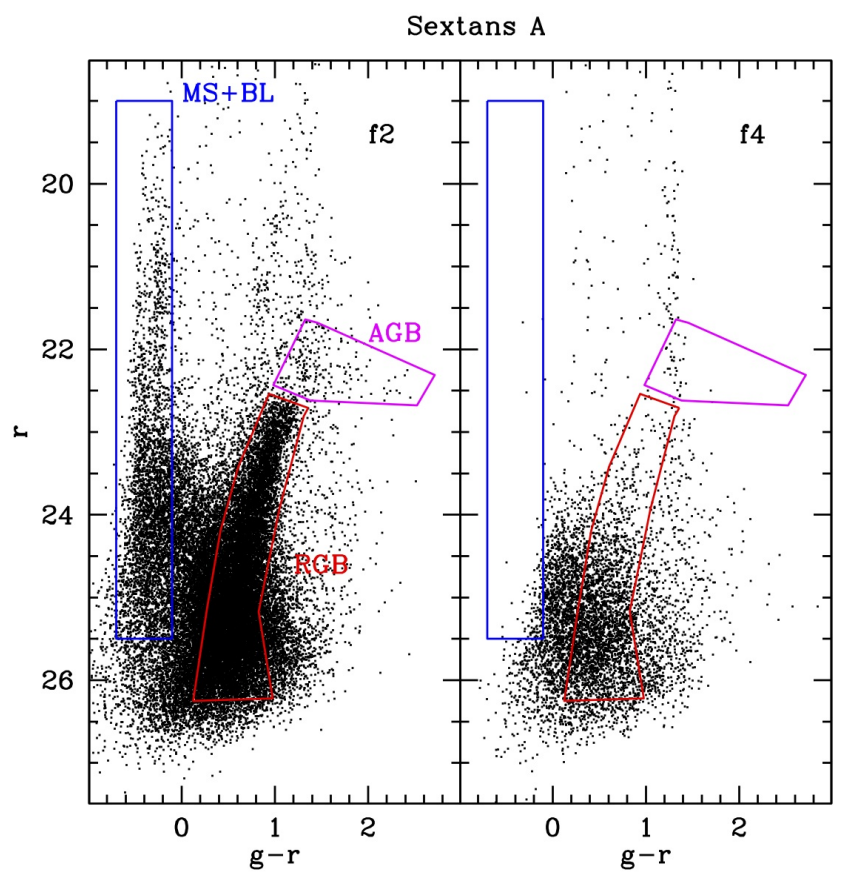

Fig. 5. Boxes adopted to select stars in different evolutionary phases are superimposed on the CMDs of a field dominated by Sex A $(f 2)$ and a field dominated by the back/foreground population in its surroundings $(f 4)$.

that the radial distributions of AGB stars presented here are the convolution of the distribution of genuine AGBs and of local contaminants that are uniformly distributed (with the additional effects of holes and gaps). As a result, the true radial distributions of AGB stars should be more centrally concentrated than is shown here. All the comparisons shown in Fig. 6 are between subsamples with the same cut at faint magnitudes, i.e, that have approximately the same degree of completeness; this condition is guaranteed for the $r<23.5$ samples (see Fig. 3).

In Fig. 6 it is apparent that in both galaxies the MS+BL population is much more concentrated than all the other stellar species considered in the comparison; in particular, they are confined in the innermost $\simeq 3^{\prime} \simeq 1.3 \mathrm{kpc}$. The effect is clearer and more robustly determined for stars brighter than $r=23.5$, for the reasons mentioned above.

Asymptotic Giant Branch stars appear as concentrated as or more concentrated than bright RGB stars, in Sex A and Sex B, respectively, in spite of the effects of contamination. Hence, both Sex A and Sex B appear to follow the general trend observed in all dwarf galaxies (see, e.g. Harbeck et al. 2001; Tolstoy et al. 2009), i.e, younger/more metal rich populations are preferentially found near the centre, while older (and more metal-poor) populations have extended distributions and dominate in the outermost regions.

\section{Structure}

In Fig. 7 we show the azimuthally averaged major-axis surface brightness (SB) profile of Sex A and Sex B. The profile was obtained by joining the $V$-band surface photometry from HE06 out to $r_{\epsilon}=3.1^{\prime}$ (for Sex A) or $r_{\epsilon}=4.7^{\prime}$ (for Sex B), with the surface density profile obtained from star counts in elliptical annuli, keeping fixed the values of $\epsilon$ and PA derived in Sect. 2.5 (see Federici et al. 2007, and Paper I for a discussion of the procedure and details). The large overlap region between the profiles from 

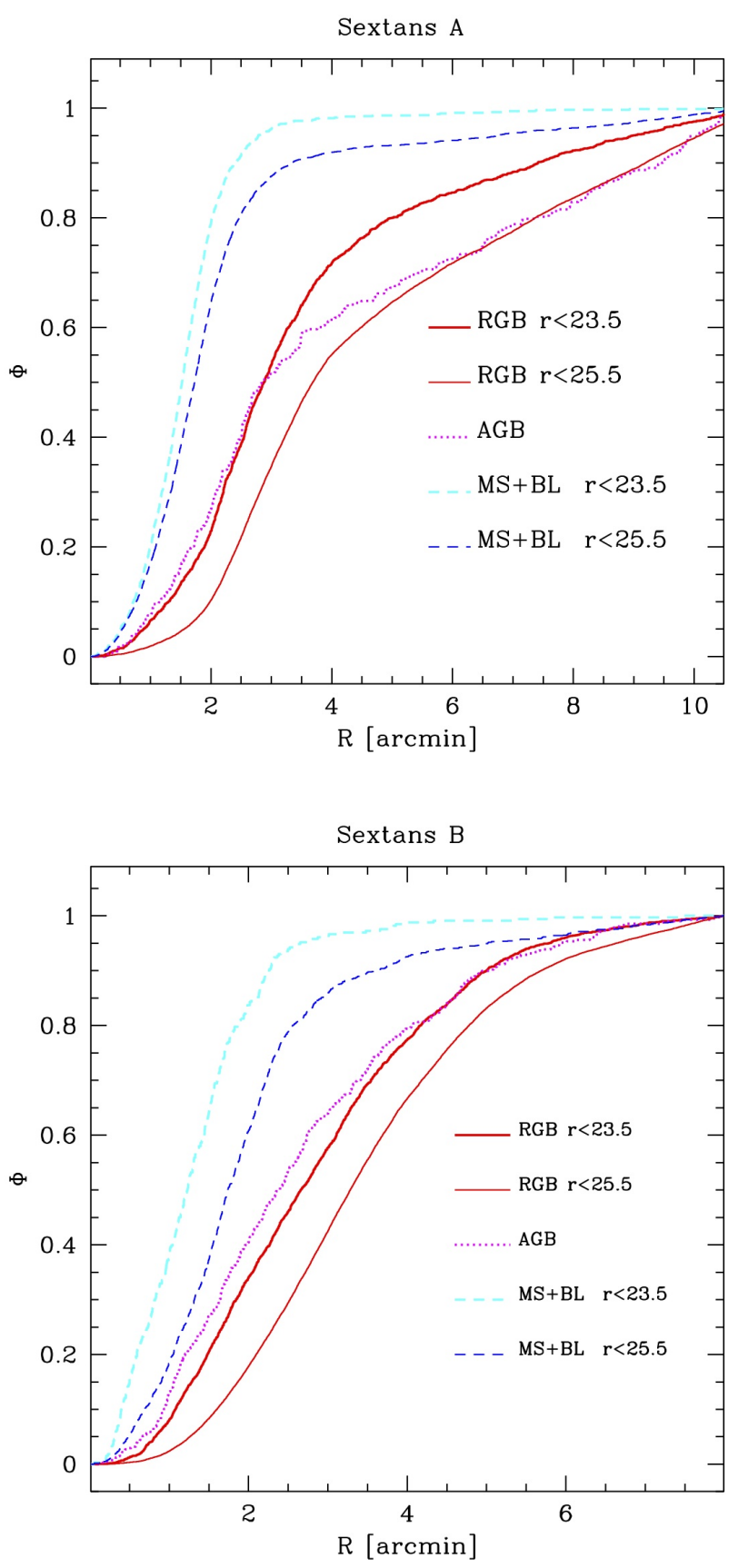

Fig. 6. Cumulative radial distribution of different stellar species, selected according to Fig. 5, for Sex A (upper panel) and Sex B (lower panel). Since this is a comparison between different populations within the same galaxy, for simplicity we consider the distributions in the circular radius $R$.

surface photometry and from star counts was used to normalise the star counts profile, shifting it to the same $V$ magnitude scale of the surface photometry profile.

For star counts, we used, as homogeneous density tracers, the candidate RGB stars enclosed in the polygonal box shown in Fig. 5, except for the innermost portion of the Sex A profile where we also included bright $(r \leq 23.0) \mathrm{MS}$ and BL stars bluer than $g-r=0.0$. The background is estimated in wide areas in the $f 4$ fields of the two samples, located as distant as possible from the centres of the galaxies. The profiles are constructed by applying bright magnitude limits to the RGB selection in the inner regions and going to fainter limits in the outskirts to get rid
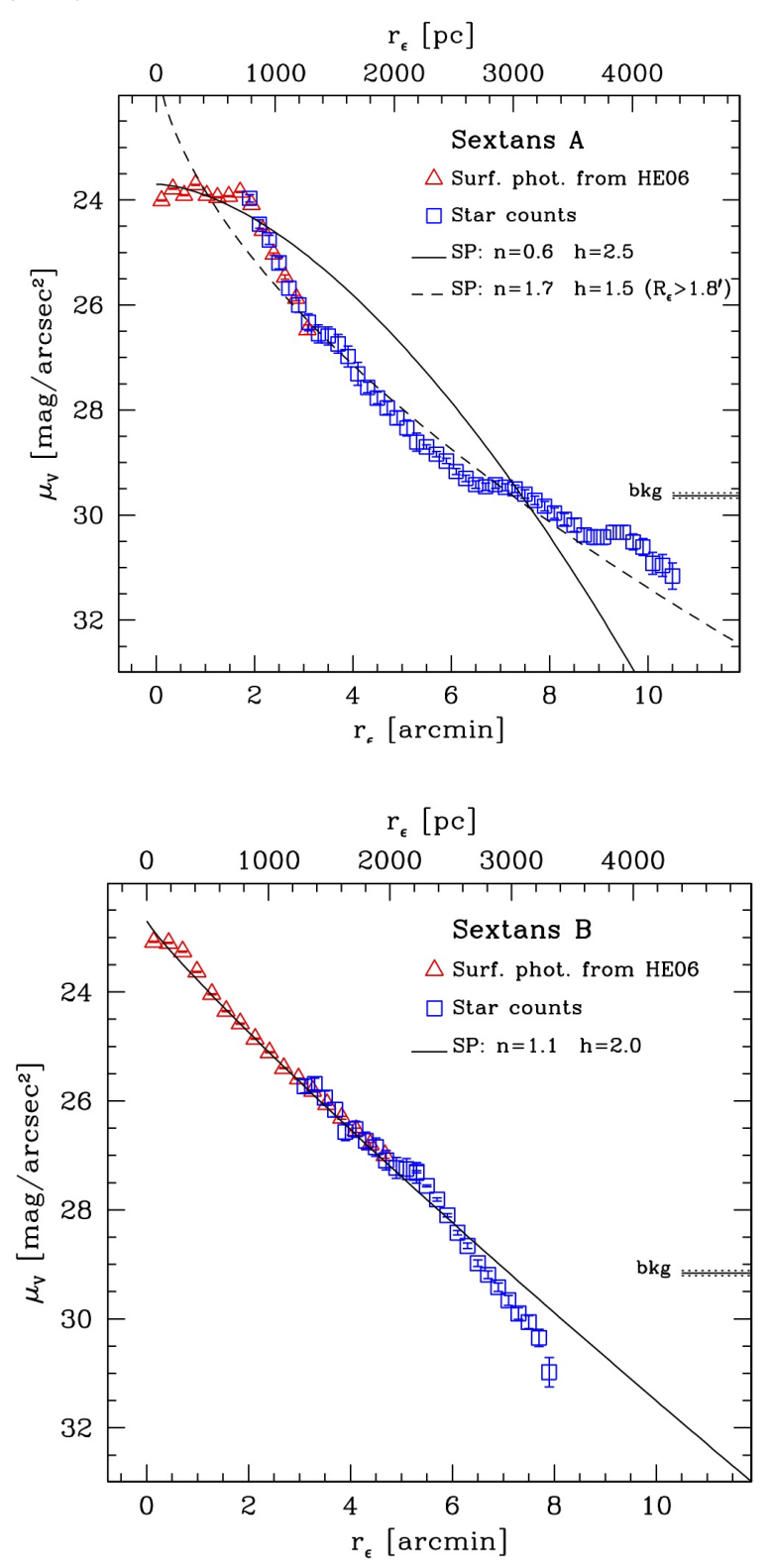

Fig. 7. Surface brightness profiles of Sex A (upper panel) and Sex B (lower panel) in $V$ band obtained by joining surface photometry from HE06 (open triangles) and star counts (open squares) profiles. The continuous lines are the Sérsic models that best fit the profiles. The dashed line in the upper panel is the Sérsic model that best fits the observed profile for $R_{\epsilon}>1.8^{\prime}$, i.e, excluding the flat portion of the profile near the centre of the galaxy. The level of the background (bkg) and the associated uncertainty are also shown.

of variations of the completeness with distance from the galaxy centre and to have the highest SB sensitivity in the low-SB external regions. The process starts by finding the upper limit in magnitude that selects a sample of tracer stars providing a profile with a large overlapping region with the surface photometry profile, where the slope of the two profiles is indistinguishable (e.g. the regions where triangles and squares overlaps in Fig. 7). This guarantees that, from the beginning of the same-slope region, there is no radial variation in completeness for the considered sample (hence, the star counts profile is reliable), and allows a robust normalisation of the star counts profile to the photometric scale of the surface photometry profile. Then star counts profiles from samples with fainter magnitude limits can be joined to the 
composite profile with the same criteria and techniques until the whole sample (RGB stars with $r \leq 26.0$, in the present case) is considered. In both galaxies, the incompleteness (and its radial variation) in the innermost regions $\left(r_{\epsilon} \leq 2^{\prime}-3^{\prime}\right)$ is too severe (see, e.g. Fig. 3) to trace the SB with star counts; this region must be covered with the SB profile from surface photometry that, by definition, is not affected by incompleteness.

As in the case of VV124 presented in Paper I, our newly derived composite profiles reaches much larger radii (by a factor of $\gtrsim 2$ ) than previous studies, which were based solely on surface photometry. In particular we are able to trace the stellar profiles out to $r_{\epsilon} \simeq 10.5^{\prime}$ and $r_{\epsilon} \simeq 8.0^{\prime}$ for Sex A and Sex B, respectively, showing that these galaxies are also much more extended than previously observed. At present, all the isolated dwarfs for which deep wide-field photometry has been acquired with $8 \mathrm{~m}$ class telescopes have been found to have SB profiles extending down to SB levels $\left(~ \gtrsim 30 \mathrm{mag} / \mathrm{arcsec}^{2}\right)$ that cannot be reached with ordinary surface photometry, at least for these nearby (resolved) galaxies (see Vansevičius et al. 2004; Sanna et al. 2010; Paper I; and this study).

The profiles of both Sex A and Sex B display discontinuities and cannot be satisfactorily fitted with a single Sérsic (1968) profile. Sex A shows a flat branch in the innermost $\simeq 1.8^{\prime}$, that is consistently traced in our own XVISTA photometry and by HE06, who classify this galaxy among the "flat inner profile" dwarfs (see HE06 for discussion). We will briefly return to this flattening in the stellar SB in Sect. 5, since it appears to have a counterpart in the H I profile.

Beyond $r_{\epsilon} \simeq 1.8^{\prime}$ the profile displays two additional changes of slope, a shallow (and possibly not significant) one coinciding with a weak kink at $r_{\epsilon} \simeq 3^{\prime}$, and a more pronounced flattening at $r_{\epsilon} \simeq 6^{\prime}$. The outer, more significant, change of slope is associated with a probable tidal tail, described in Sect. 4.1 below. The observed profile has some similarity with that of another isolated dIrr that has been traced down to very low SB, Leo A (Vansevičius et al. 2004). We note that a single Sérsic model ${ }^{7}$ is significantly inadequate to represent the observed profile, even if the flat inner region is excluded from the fit.

The profile of Sex B displays a single change of slope, a weak steepening at $r_{\epsilon} \simeq 5^{\prime}$. Breaks in the SB profile are common in the outer region of disc galaxies (van der Kruit 1979, HE06). According to Pohlen \& Trujillo (2006), the downbending profile with the steeper outer region (their Type II), as we observe in Sex B, is very common in late-type spiral galaxies (see Ferguson et al. 2007, for a local example); the incidence of the class declines for earlier types. The position $(\sim 2.5$ scalelength) of the profile break observed in Sex B is slightly more external than the typical values for Type II disc galaxies $(\sim 2.0$ scalelength Pohlen \& Trujillo 2006, HE06).

Since these galaxies should have the broad structure of fluffy thick discs, it is clear that the inclination of their principal plane with respect to the line of sight should play a role in determining the shape of their SB profile as seen from the Earth. Unfortunately the inclination of both galaxies is poorly constrained by H I data (see Sect. 5). Moreover, it is not at all guaranteed that the main stellar bodies of the galaxies (especially the backbones of old stars) have the same morphology and/or inclination of the $\mathrm{HI}$ disc.

\footnotetext{
7 Here we adopt the formalism by Ciotti \& Bertin (1999), with the SB profile given by $I(r)=I_{0} \mathrm{e}^{-b \eta^{\frac{1}{n}}}$ (their Eq. (1)), with $\eta=\frac{r}{h}, h$ being the half-light radius. The value of the constant $b$ is from their Eq. (25), which works well for $n \gtrsim 1.0$.
}

Table 4. Observed surface brightness profiles.

\begin{tabular}{|c|c|c|c|c|c|}
\hline \multicolumn{3}{|c|}{ Sex A } & \multicolumn{3}{|c|}{ Sex B } \\
\hline $\begin{array}{l}r_{\epsilon} \\
\operatorname{arcmin}\end{array}$ & $\begin{array}{c}\mu_{V} \\
\mathrm{mag} / \operatorname{arcsec}^{2}\end{array}$ & Source & $\begin{array}{l}r_{\epsilon} \\
\operatorname{arcmin}\end{array}$ & $\begin{array}{c}\mu_{V} \\
\mathrm{mag} / \operatorname{arcsec}^{2}\end{array}$ & Source \\
\hline 0.11 & $24.01 \pm 0.01$ & HE06 & 0.14 & $23.08 \pm 0.01$ & HE06 \\
\hline 0.34 & $23.79 \pm 0.01$ & HE06 & 0.43 & $23.10 \pm 0.01$ & HE06 \\
\hline 0.57 & $23.91 \pm 0.01$ & HE06 & 0.71 & $23.26 \pm 0.01$ & HE06 \\
\hline 0.80 & $23.71 \pm 0.01$ & HE06 & 0.99 & $23.63 \pm 0.01$ & HE06 \\
\hline 1.03 & $23.91 \pm 0.01$ & HE06 & 1.28 & $24.04 \pm 0.01$ & HE06 \\
\hline 1.25 & $23.95 \pm 0.01$ & HE06 & 1.56 & $24.35 \pm 0.01$ & HE06 \\
\hline 1.48 & $23.93 \pm 0.01$ & HE06 & 1.84 & $24.58 \pm 0.01$ & HE06 \\
\hline 1.71 & $23.85 \pm 0.01$ & HE06 & 2.13 & $24.86 \pm 0.01$ & HE06 \\
\hline 1.90 & $23.97 \pm 0.07$ & $\mathrm{sc}$ & 2.41 & $25.11 \pm 0.01$ & HE06 \\
\hline 2.10 & $24.46 \pm 0.08$ & $\mathrm{sc}$ & 2.69 & $25.40 \pm 0.01$ & HE06 \\
\hline 2.30 & $24.76 \pm 0.09$ & $\mathrm{sc}$ & 2.98 & $25.59 \pm 0.01$ & HE06 \\
\hline 2.50 & $25.20 \pm 0.11$ & $\mathrm{sc}$ & 3.10 & $25.73 \pm 0.12$ & $\mathrm{sc}$ \\
\hline 2.70 & $25.68 \pm 0.13$ & $\mathrm{sc}$ & 3.30 & $25.69 \pm 0.11$ & $\mathrm{sc}$ \\
\hline 2.90 & $26.00 \pm 0.14$ & $\mathrm{sc}$ & 3.50 & $25.94 \pm 0.12$ & $\mathrm{sc}$ \\
\hline 3.10 & $26.33 \pm 0.16$ & $\mathrm{sc}$ & 3.70 & $26.16 \pm 0.13$ & $\mathrm{sc}$ \\
\hline 3.30 & $26.55 \pm 0.17$ & $\mathrm{sc}$ & 3.90 & $26.57 \pm 0.16$ & $\mathrm{sc}$ \\
\hline 3.50 & $26.59 \pm 0.17$ & $\mathrm{sc}$ & 4.10 & $26.52 \pm 0.15$ & $\mathrm{sc}$ \\
\hline 3.70 & $26.74 \pm 0.18$ & $\mathrm{sc}$ & 4.30 & $26.73 \pm 0.16$ & $\mathrm{sc}$ \\
\hline 3.90 & $26.98 \pm 0.20$ & $\mathrm{sc}$ & 4.50 & $26.85 \pm 0.17$ & $\mathrm{sc}$ \\
\hline 4.10 & $27.31 \pm 0.22$ & $\mathrm{sc}$ & 4.70 & $27.09 \pm 0.18$ & $\mathrm{sc}$ \\
\hline 4.30 & $27.57 \pm 0.10$ & $\mathrm{sc}$ & 4.90 & $27.23 \pm 0.19$ & $\mathrm{sc}$ \\
\hline 4.50 & $27.77 \pm 0.11$ & $\mathrm{sc}$ & 5.10 & $27.25 \pm 0.19$ & $\mathrm{sc}$ \\
\hline 4.70 & $27.96 \pm 0.12$ & $\mathrm{sc}$ & 5.30 & $27.32 \pm 0.19$ & $\mathrm{sc}$ \\
\hline 4.90 & $28.15 \pm 0.13$ & $\mathrm{sc}$ & 5.30 & $27.30 \pm 0.02$ & $\mathrm{sc}$ \\
\hline 5.10 & $28.34 \pm 0.15$ & $\mathrm{sc}$ & 5.50 & $27.56 \pm 0.02$ & $\mathrm{sc}$ \\
\hline 5.30 & $28.61 \pm 0.17$ & $\mathrm{sc}$ & 5.70 & $27.81 \pm 0.03$ & $\mathrm{sc}$ \\
\hline 5.50 & $28.70 \pm 0.04$ & $\mathrm{sc}$ & 5.90 & $28.10 \pm 0.03$ & $\mathrm{sc}$ \\
\hline 5.70 & $28.84 \pm 0.05$ & $\mathrm{sc}$ & 6.10 & $28.42 \pm 0.04$ & $\mathrm{sc}$ \\
\hline 5.90 & $28.97 \pm 0.05$ & $\mathrm{sc}$ & 6.30 & $28.66 \pm 0.05$ & $\mathrm{sc}$ \\
\hline 6.10 & $29.17 \pm 0.06$ & $\mathrm{sc}$ & 6.50 & $28.98 \pm 0.06$ & $\mathrm{sc}$ \\
\hline 6.30 & $29.30 \pm 0.06$ & $\mathrm{sc}$ & 6.70 & $29.19 \pm 0.07$ & $\mathrm{sc}$ \\
\hline 6.50 & $29.42 \pm 0.07$ & $\mathrm{sc}$ & 6.90 & $29.42 \pm 0.08$ & $\mathrm{sc}$ \\
\hline 6.70 & $29.46 \pm 0.07$ & $\mathrm{sc}$ & 7.10 & $29.66 \pm 0.09$ & $\mathrm{sc}$ \\
\hline 6.90 & $29.42 \pm 0.07$ & $\mathrm{sc}$ & 7.30 & $29.90 \pm 0.11$ & $\mathrm{sc}$ \\
\hline 7.10 & $29.47 \pm 0.07$ & $\mathrm{sc}$ & 7.50 & $30.06 \pm 0.12$ & $\mathrm{sc}$ \\
\hline 7.30 & $29.50 \pm 0.07$ & $\mathrm{sc}$ & 7.70 & $30.35 \pm 0.16$ & $\mathrm{sc}$ \\
\hline 7.50 & $29.60 \pm 0.08$ & $\mathrm{sc}$ & 7.90 & $30.98 \pm 0.27$ & $\mathrm{sc}$ \\
\hline 7.70 & $29.72 \pm 0.08$ & $\mathrm{sc}$ & & & \\
\hline 7.90 & $29.83 \pm 0.09$ & $\mathrm{sc}$ & & & \\
\hline 8.10 & $29.96 \pm 0.10$ & $\mathrm{sc}$ & & & \\
\hline 8.30 & $30.08 \pm 0.11$ & $\mathrm{sc}$ & & & \\
\hline 8.50 & $30.19 \pm 0.12$ & $\mathrm{sc}$ & & & \\
\hline 8.70 & $30.38 \pm 0.13$ & $\mathrm{sc}$ & & & \\
\hline 8.90 & $30.42 \pm 0.14$ & $\mathrm{sc}$ & & & \\
\hline 9.10 & $30.42 \pm 0.14$ & $\mathrm{sc}$ & & & \\
\hline 9.30 & $30.33 \pm 0.13$ & $\mathrm{sc}$ & & & \\
\hline 9.50 & $30.33 \pm 0.13$ & $\mathrm{sc}$ & & & \\
\hline 9.70 & $30.51 \pm 0.15$ & $\mathrm{sc}$ & & & \\
\hline 9.90 & $30.61 \pm 0.16$ & $\mathrm{sc}$ & & & \\
\hline 10.10 & $30.92 \pm 0.21$ & $\mathrm{sc}$ & & & \\
\hline 10.30 & $30.96 \pm 0.21$ & $\mathrm{sc}$ & & & \\
\hline 10.50 & $31.16 \pm 0.25$ & $\mathrm{sc}$ & & & \\
\hline
\end{tabular}

Notes. HE06 = surface photometry from HE06; sc = star counts from the present analysis. The uncertainties in the HE06 photometry have been approximated at the 0.01 mag level.

Looking at Fig. 4 above, it can be appreciated that no obvious change in the stellar content occurs at the profile breaks in Sex A or Sex B, as anticipated in Sect. 3.

Integrating the profiles shown in Fig. 7 numerically we obtain integrated $V$ magnitudes $V_{\text {tot }}=11.7 \pm 0.3$ and $V_{\text {tot }}=$ $11.7 \pm 0.2$, and half-light radii $r_{\mathrm{h}}=1.8^{\prime}$ and $r_{\mathrm{h}}=1.9^{\prime}$, for Sex A 
and Sex B, respectively, in reasonable agreement with the values reported in the literature (see, e.g. HE06, M12), which are based on much less extended profiles and/or fits with (single) exponential profiles.

\subsection{Density maps}

In Fig. 8 we present the density maps for Sex A and Sex B obtained from RGB star counts on a fixed regular grid of nodes spaced by $0.1^{\prime}$, with the same adaptive algorithm described in Paper $\mathrm{I}^{8}$. The lightest shade of grey corresponds to an overdensity at $3 \sigma$ above the background level (estimated in $\mathrm{f} 4$, as described in Sect. 4), and it is the outermost density contour that we can reliably trace with our data. Single isolated weak peaks with typical scales $\lesssim 1^{\prime}$ can correspond to clusters of galaxies whose red sequence galaxies fall in the RGB selection box. The small blob in the lower right corner of the northern chip in the Sex B map is a good example that can be easily identified in the original images. However these features are very small compared to the contours of the galaxies and never exceed $5 \sigma$ above the background level, hence they cannot have a significant impact on the morphology of Sex A and Sex B as displayed in Fig. 8. All the RGB stars selected for the analysis are also plotted in the maps to give an idea of the sampling bias that are obviously affecting them (inter-chip gaps, saturated stars, etc.). In this case the overall view of the main galactic bodies we are interested in is also not compromised by these small-scale effects (although the discontinuities due to the gaps are clearly evident). Finally, the apparent density decline in the central region of the galaxies is due to the higher degree of crowding, and consequently lower completeness (see Sect. 2.4); in the case of Sex A the bright foreground star TYC 4907-713-19 also plays a role in preventing the detection of any star over a region of several arcsec. Again this is not a serious problem in the present context since the density in the central part is well described by the azimuthally averaged profiles, while here we are especially interested in the morphology of the most external regions of our galaxies.

The map of Sex A reveals that the denser roundish main body is surrounded by a vast low-density and elongated envelope, where the density declines very gently. The feature is also clearly visible in the $10 \sigma$ contours and so there is no doubt that it is real. It is very interesting to note that the transition between the central body and the envelope occurs in correspondence with the outermost change of slope in the profile, at $R \simeq 6^{\prime}$. The broad S-shape of the outer envelope and its correspondence with a break toward a shallower slope in the density profile are suggestive of a tidal origin (Johnston et al. 1999; Peñarrubia et al. 2008; Muñoz et al. 2008). In both maps in Fig. 8 we report the direction to the nearest dwarf (Sex B, in this case) and to NGC 3109, the most massive member of the association Sex A and Sex B belong to. No obviously significant alignment is apparent, but it must be reminded that the orientation of tidal tails depends significantly on the orbital phase (Klimentowski et al. 2009).

At odds with its twin, Sex B displays a remarkably elliptical but compact and regular morphology even in its outermost regions. Since the masses of the two galaxies are similar (see

\footnotetext{
8 In Fig. 10 we show the stellar density maps obtained with the matched filter technique (see Rockosi et al. 2002) that was also used in Paper I. As done in Paper I, we present the maps obtained with both methods, as their full agreement lends further support to the reliability of the discussed features.

9 A $V=11.5$ star located just 77.5 " to the NE of the Sex A centre, that is heavily saturated in our images (see Fig. 1).
}
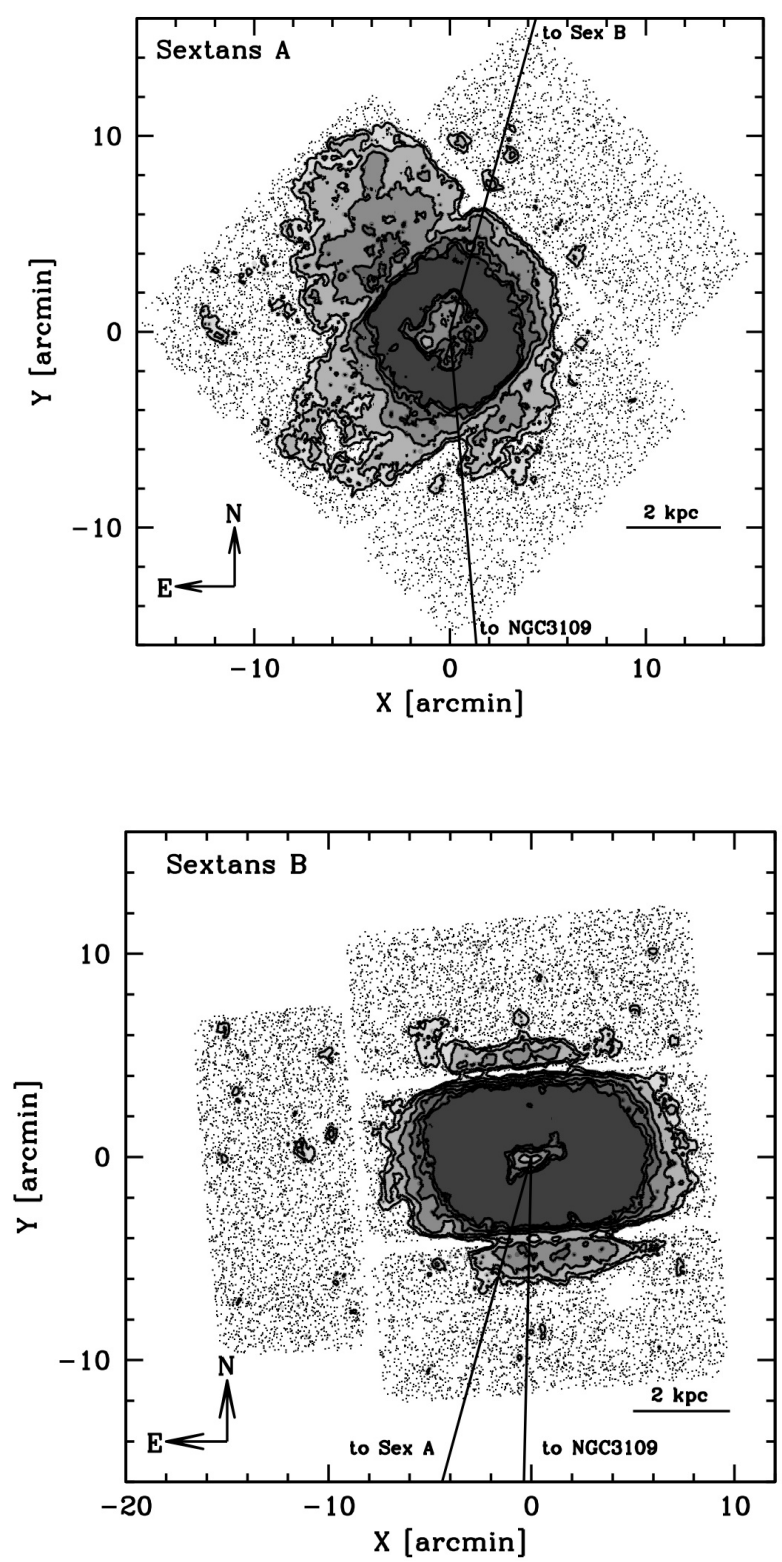

Fig. 8. Stellar surface density contour maps from RGB counts. The contours/levels of grey correspond to a density of $3,5,10,20$, and $40 \sigma$ above the background, from the lightest to the darkest shade of grey. The density depressions near the centre of the main galactic bodies are due to the low degree of completeness in the very crowded innermost regions. The directions to nearby galaxies of the NGC 3109 group are plotted as continuous lines. The distances to NGC 3109 are $\sim 500 \mathrm{kpc}$ and $\sim 700 \mathrm{kpc}$, for Sex A and Sex B, respectively; the distance between Sex A and Sex B is $\sim 250 \mathrm{kpc}$. All RGB stars are also plotted (as dots) to provide a direct illustration of the effects of the footprint of the camera and/or heavily saturated foreground stars.

Sect. 5) the different morphology can be accounted for only by different initial conditions or different orbital histories, i.e, if Sex A was more strongly affected than Sex B by a past tidal interaction (see Sect. 6).

We will discuss in more detail the hypothesis of tidal interactions in the NGC 3109 group, also in relation with the results by Penny et al. (2012) and Bellazzini et al. (2013) in Sect. 6. 


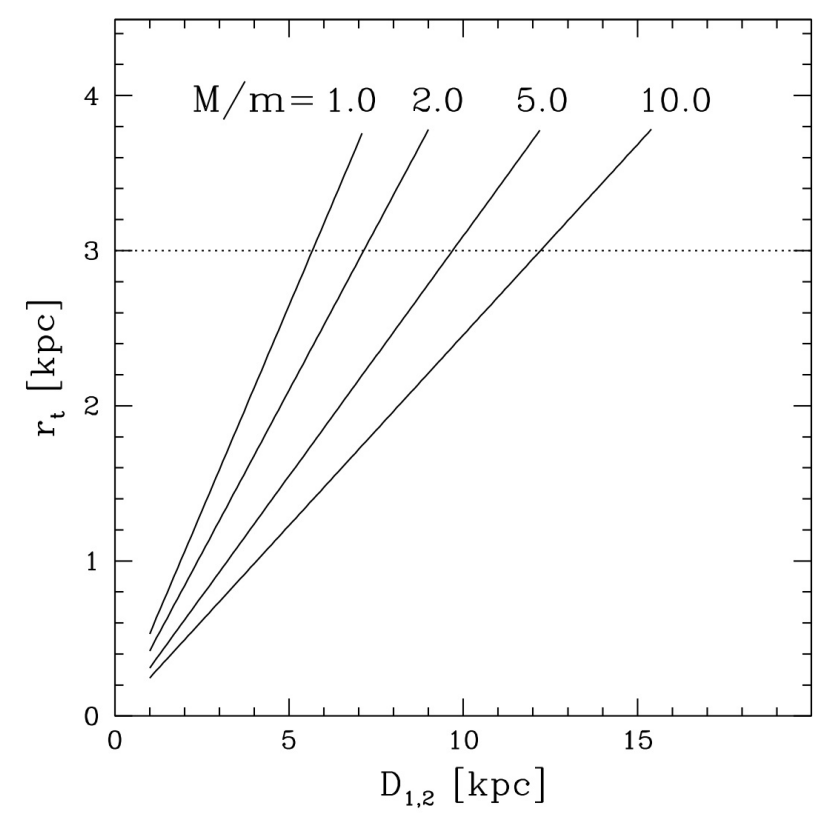

Fig. 9. Tidal radius as a function of mutual distance $\left(D_{1,2}\right.$ between two galaxies of various mass ratios $(\mathrm{M} / \mathrm{m})$, from Eq. (2). The dotted line marks the approximate tidal radius of Sex A and Sex B, the range of $M / m$ considered in the figure corresponds to the mass ratios between Sex A and Sex B, and between Sex A/Sex B and NGC 3109.

Here, as in Paper I, we use a simple equation for the tidal radius (Keenan 1981a,b),

$r_{\mathrm{t}}=\frac{2}{3}\left(\frac{m}{2 M}\right)^{\frac{1}{3}} D_{1,2}$

which is appropriate for a logarithmic potential (see Bellazzini et al. 2004, for discussion and references) to obtain some basic constraint on the encounter that can be at the origin of the putative tidal tail of Sex A. The symbols $m$ and $M$ are the mass of the involved bodies (with $M>m$ ) and $D_{1,2}$ is their mutual distance.

Assuming $\simeq 3 \mathrm{kpc}$ as the approximate truncation radius for this galaxy (from Fig. 7) we show in Fig. 9 the perigalactic distance at which this limit can be set by tides for various values of the mass ratio of the interacting galaxies. In the range of mass ratios characteristic of an encounter with Sex B $(M / m=1-2)$ a passage in the range $5 \mathrm{kpc} \lesssim D_{1,2} \lesssim 8 \mathrm{kpc}$ is required, while for encounters with NGC 3109 a passage at $10 \mathrm{kpc} \lesssim D_{1,2} \lesssim 13 \mathrm{kpc}$ will be sufficient. We note that Sex A is $\simeq 250 \mathrm{kpc}$ from Sex B and $\simeq 508 \mathrm{kpc}$ from NGC 3109 (see Bellazzini et al. 2013), and so at present it is essentially undisturbed by the tides of its closest companions. For $M / m \geq 1000$, corresponding to the mass ratio between Sex A/Sex B and the MW, the $r_{t}=3 \mathrm{kpc}$ line is matched at $D_{1,2} \geq 57 \mathrm{kpc}$, similar to the peri-galactic distance of the Magellanic Clouds, according to the most recent analysis (Kallivayalil et al. 2013).

\section{Neutral hydrogen}

We have analysed the distribution and kinematics of the neutral hydrogen using the publicly available deep observations of Sex A and Sex B provided by the VLA-ANGST (Ott et al. 2012) ${ }^{10}$ survey.

In Fig. 10 we superimpose the H I surface density contours to the stellar surface density maps we obtained from star counts

${ }^{10}$ https://science.nrao.edu/science/surveys/vla-angst/ with the matched filter technique (see Paper I). There are a few interesting conclusions that can be drawn from this simple comparison:

- In both galaxies the neutral hydrogen component is comparably (or less) extended than the distribution of the stars (see also Fig. 11). It was previously noted that this is also the case in VV124 (Paper I) but that galaxy has a ratio of $\mathrm{HI}$ mass to stellar mass $M_{\mathrm{HI}} / M_{*} \simeq 0.1$, while Sex A and Sex B have $M_{\mathrm{HI}} / M_{*} \gtrsim 1.0$ (adopting $\frac{M}{L_{V}}=1.0$, in solar units, for the stellar component), i.e, they are much more gas-rich than VV124. It must be noted that the truncation of the H I distribution does not necessarily imply that no bound gas is present at larger radii, since extended ionised gas of very low surface brightness is expected to surround the H I discs of spirals and irregulars (Maloney 1992; Corbelli \& Salpeter 1993).

- The outer contours of the gas and the star surface density distributions have remarkably similar shapes in Sex B. The same is true for the main body of Sex A. In general the overall large-scale correlation between the gas and the stars is quite high. This was far from obvious, since the stellar maps are based on old-intermediate age tracers. On the other hand, the correlation is very strong between the highest density peaks of the $\mathrm{HI}$ and the youngest stars and associations, as expected.

- The H I distribution in Sex A seems to end near the $r_{\epsilon} \simeq 6^{\prime}$ break in the SB profile, marking the emergence of the wide elongated halo surrounding the galaxy. This supports the interpretation of this structure as a tidal tail. A tidal interaction would strain and strip stars and gas in equal measure, but the stripped gas would be easily dissipated by ram pressure (also induced by the corona of gas ejected from the group members; see Shen et al. 2014) while the stars can remain partially bound, or unbound but on orbits very similar to the main body of the galaxy, for a long time. The outer H I warp is also compatible with some tidal disturbance in the past.

In Fig. 11 we compare the H I and SB profiles. It is interesting to note that the inner flattening of the SB profile in Sex A corresponds to a central drop in the H I column density, possibly suggesting that the recent strong star formation activity may have a role in the formation of a constant SB core (see, e.g. Teyssier et al. 2013, and references therein). A similar correspondence, on much smaller scales, may be also present in the profile of Sex B, for $r_{\epsilon} \lesssim 1.0^{\prime}$.

We have performed tilted ring fitting of the velocity fields and built some 3D models of the datacubes using the standard GIPSY software (see e.g. Battaglia et al. 2006). We found that in both galaxies the kinematics is clearly dominated by rotation although some non-circular motions may be present. Both Sex A and Sex B show nearly solid-body rotation with some indication of flatting in the outer parts. The kinematic position angles of the discs are well determined within a few degrees and are $96^{\circ}$ and $61^{\circ}$ for Sex A and B, respectively. For Sex A this refers to the inner disc while in the outer parts there are signs of a warp and a change in the position angle towards lower values.

Unfortunately, the inclination angles are very difficult to constrain using the HI datacubes as often happens for solid body rotation. Acceptable fits can be obtained with a wide range of inclinations provided that the thickness of the disc changes accordingly. For instance, acceptable models for Sex B are obtained with inclinations spanning from $20^{\circ}$ to almost $80^{\circ}$. In the case of $i=20^{\circ}$, the disc can be quite thin with (exponential) scaleheight lower than $300 \mathrm{pc}$, while if one assumes 80 degrees the 

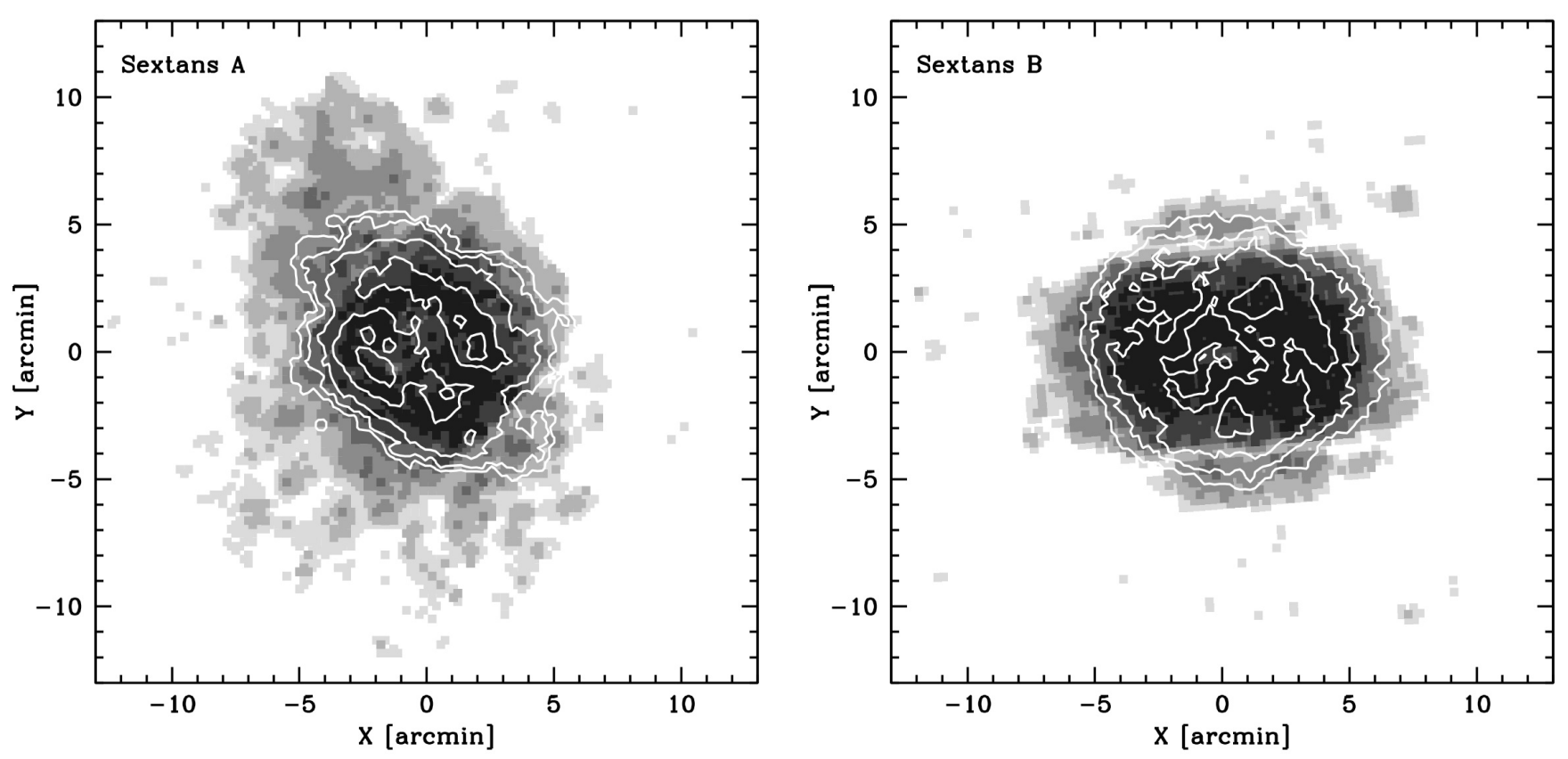

Fig. 10. H I surface density contours (white) derived from Ott et al. (2012) maps are superimposed onto the stellar density maps obtained with the matched filter technique (greyscale). Contour levels are $3,9,27,81,240$, and $720 \times 10^{19} \mathrm{~cm}^{-2}$. The levels of grey correspond to density of $3,5,10$, 20,40 , and $80 \sigma$ above the background, from the lightest to the darkest shade of grey.

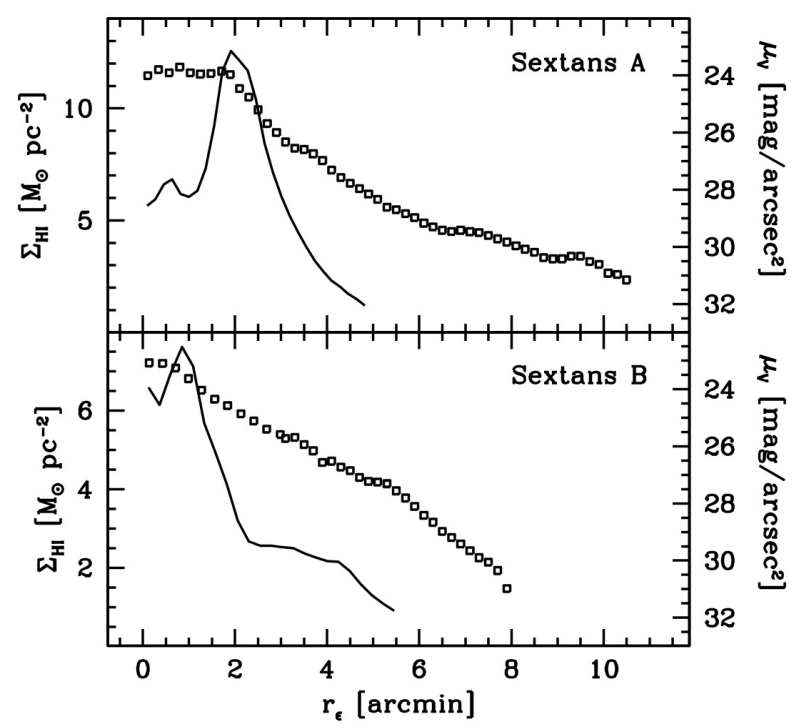

Fig. 11. H I surface density profiles (continuous lines, scale on the left vertical axis) compared to surface brightness profiles (empty squares, scale on the right vertical axis), for Sextans A (upper panel) and Sextans B (lower panel).

required scale-height is about $1 \mathrm{kpc}$. If we assume an intermediate value of $i=40$ degrees, the rotation at the last measured point is $v_{\text {last }} \sim 50 \mathrm{~km} \mathrm{~s}^{-1}$.

In the case of Sex A, the inclination is also rather unconstrained by the HI kinematics although it is clearly different from zero given the rotation pattern observed. The overall morphology of the HI and of the stellar body suggests a relatively low inclination. If we assume $i=20^{\circ}$ we obtain a $v_{\text {last }} \sim 50 \mathrm{~km} \mathrm{~s}^{-1}$ for this galaxy as well.

Under these assumptions, the dynamical masses estimated at the last measured points of the rotation curves would be of about
$1 \times 10^{9} M_{\odot}$, implying a mass-to-light ratio $\frac{M}{L_{V}} \sim 25$ and a dark-tobaryonic mass ratio of $\sim 10$. We stress that these values are very uncertain given the low inclinations of the galaxies; for instance, an inclination as low as $i=20^{\circ}$ for Sextans B would lead to a dynamical mass four times larger.

\section{Summary and discussion}

We present new deep wide-field photometry, and a re-analysis of existing $\mathrm{H}$ I data for the two isolated dwarf irregular galaxies Sextans A and Sextans B, members of the NGC 3109 association of dwarfs (Tully et al. 2006; Bellazzini et al. 2013). The main results of the present study can be summarised as follows:

- In both galaxies there are obvious population gradients on a global scale. As generally observed in other dwarfs, younger stars are confined to the innermost regions while older and older stars have more and more extended distributions. The mean metallicity of the old stellar component is low $(\langle[\mathrm{Fe} / \mathrm{H}]\rangle \lesssim-1.4)$ with no obvious metallicity gradient. We provide the first estimate of the mean metallicity for the oldest stellar population of Sex B. Using the colour distribution of bright RGB stars from HST-ACS photometry we obtain $\langle[\mathrm{Fe} / \mathrm{H}]\rangle_{\mathrm{SexB}}=-1.6$.

- One candidate compact star cluster has been identified in Sex A and two in Sex B, in addition to the already confirmed C1 in Sex B (Sharina et al. 2007).

- We extended the existing SB profiles from surface photometry (reaching $\mu_{V} \sim 27.5 \mathrm{mag} / \mathrm{arcsec}^{2}$ ) with profiles from star counts reaching $\mu_{V} \sim 31 \mathrm{mag} / \operatorname{arcsec}^{2}$. Both galaxies display SB profiles that are not adequately reproduced by a single Sérsic (1968) model. The profile of Sex B shows a downbending at $r_{\epsilon} \simeq 5^{\prime}$, while that of Sex A presents a flat central region (for $r_{\epsilon} \simeq 1.8^{\prime}$, strongly correlated with a drop in the H I surface density profile), and an upward bending at $r_{\epsilon} \simeq 6^{\prime}$. 
- We show that the stellar body of the two galaxies is much more extended than previously traced with surface photometry. In particular we can trace the profiles of Sex A and Sex B out to $\sim 4 \mathrm{kpc}$ and $\sim 3.5 \mathrm{kpc}$, respectively (w.r.t $\lesssim 2 \mathrm{kpc}$ ). In both cases the stellar body appears to be more extended than the H I disc.

- The kinematics of the HI are dominated by a significant solid-body rotation. The large uncertainties on the inclinations of the discs prevents an accurate determination of the maximum rotational velocity. Under conservative assumptions we find $v_{\text {last }} \simeq 50 \mathrm{~km} \mathrm{~s}^{-1}$ for both galaxies, corresponding to a dynamical mass of $\sim 10^{9} \mathrm{M}_{\odot}$.

- The stellar density map of Sex A shows that the roundish main body of the galaxy, nicely matched by the H I disc in position and shape, is surrounded by a flatter and elongated LSB halo of stars. This structure appears to display a broad S-shape and its onset corresponds to the outer up-bending change in slope of the SB profile. Both features are typical of tidal tails (Johnston et al. 1999; Peñarrubia et al. 2009; Muñoz et al. 2008) and the structure and kinematics of the HI, with a warp in correspondence with the position of the tail, are also fully compatible with the hypothesis of a tidal origin for the observed structure. It is very interesting to note that a similar feature has been recently identified in another member of the same association Sex A belongs to, the Antlia dIrr, and it has been interpreted as a tidal tail due to a past interaction with NGC 3109 (Penny et al. 2012).

- Basic considerations on the size and masses of the involved galaxies suggests that both an interaction with another member of the NGC 3109 association or with the Milky Way are plausible as a possible origin for the putative tidal tail of Sex A. In either case, the comparison between the relative distances and velocities indicates that these interactions should have happened several Gyr ago (see below). This may appear to be a problem for the tidal interpretation, since tidal tails are not expected to be very long-lived. However, it must be noted that the very low SB portions of the tails can persist for long times (see Peñarrubia et al. 2009, e.g. their Fig. 2), and that, in general, available theoretical studies have considered the case of dwarfs orbiting a main galaxy, while in the present case it is likely that we are dealing with a single close encounter (possibly between dwarfs) followed by an evolution in (progressively deeper) isolation. Dedicated $\mathrm{N}$ body simulations are probably needed to adequately explore this specific case.

The last point should be considered in the context of the past history of the NGC 3109 association. Teyssier et al. (2012) compared the observed distances and velocities of LG dwarfs with those of state-of-the-art $N$-body simulations, to deduce their past orbital histories. In their analyses, from a sample of 39 relatively isolated dwarfs, they identified ten galaxies as very likely backsplashers, i.e, stellar systems that have passed within 0.5 virial radii of a dominant member of the LG (MW or M31) in the past. Among these ten, they list NGC 3109, Antlia, Sex A, and Sex B, i.e, all the members of the NGC 3109 association that were known at the time of the Teyssier et al. (2012) analysis ${ }^{11}$. In their independent attempt to reconstruct the past path of all the LG galaxies, Shaya \& Tully (2013) also envisage a passage at $\sim 25 \mathrm{kpc}$ from the MW of most of the members of the association that occurred $\sim 7$ Gyr ago.

\footnotetext{
11 The newly discovered Leo P galaxy (Giovanelli et al. 2013) is likely a new member of the association; see Bellazzini et al. (2013, and references therein).
}

The hypothesis of a close encounter of the NGC 3109 association with the MW provides a natural scenario to explain the recent finding that all the members of the association lie within $\sim 100 \mathrm{kpc}$ (rms) of a line in space that is $\sim 1.1 \mathrm{Mpc}$ long, from one extreme (NGC 3109) to the other (Leo P), and display a gradient between velocity in the $\mathrm{LG}$ reference frame $\left(V_{\mathrm{LG}}\right)$ and distance along the line of $43 \mathrm{~km} \mathrm{~s}^{-1} \mathrm{Mpc}^{-1}$, with a rms of just $17 \mathrm{~km} \mathrm{~s}^{-1}$ (Bellazzini et al. 2013). It is interesting to note that the timescale for the association to reach the current distance from the MW (the ratio between this distance and the mean velocity of the association in the Galactic reference frame, $\sim 7 \mathrm{Gyr}$ ) is similar to the timescale to scatter by $100 \mathrm{kpc}$ from the line by the effect of random motions of $17 \mathrm{~km} \mathrm{~s}^{-1}$ ( $\left.\sim 6 \mathrm{Gyr}\right)$. This suggests that, if the association had a close encounter with the MW a few Gyr ago, at that epoch its members were very close together and/or tightly clustered in a filamentary configuration which was dynamically cold, otherwise they would be much more scattered in space at the present epoch. On the other hand, no obvious signature of this interaction can be identified in the SFHs of the members of the association presented by Weisz et al. (2011), except perhaps for Antlia, whose star formation rate peaked $\sim 6$ Gyr ago. However, SF enhancements on scales shorter than $\sim 1$ Gyr can be easily wiped out by the low temporal resolution ( $\sim 3-4 \mathrm{Gyr})$ of the reconstructed SFHs in that range of look-back times.

Given all the above arguments, in a Newtonian dynamics and $\Lambda$ - cold dark matter framework (Barkana \& Loeb 2001), it is plausible to think that the members of the NGC 3109 association, after decoupling from the Hubble flow, were originally falling toward the Milky Way along one of the thin and cold cosmological filaments that are predicted by the current paradigm of galaxy formation (Dekel et al. 2009; Lovell et al. 2011) . $^{12}$. The close encounter with the Galaxy tidally stretched the dwarfs into their present extremely elongated and dynamically cold configuration, also producing (or enhancing) the observed velocity gradient, and finally pulling all of them out of the LG (Shaya $\&$ Tully 2013). The peri-Galactic passage was not only the time when the tidal strain of the MW was maximum on each individual member of the association, but also the epoch when the members were most closely packed together, maximizing the effects and the probability of dwarf-to-dwarf interactions. It seems likely that the tidal tails of Antlia and Sex A, as well as the warp and substructures observed in NGC 3109 (Hidalgo et al. 2008; Barnes \& de Blok 2001; Dalcanton et al. 2009) were produced during that relatively short epoch of multiple interactions.

Acknowledgements. We acknowledge the support from the LBT-Italian Coordination Facility for the execution of observations, data distribution and reduction. M.B and F.F. acknowledge financial support from PRIN MIUR 20102011 project "The Chemical and Dynamical Evolution of the Milky Way and Local Group Galaxies", prot. 2010LY5N2T. We are grateful to D. Hunter for providing the surface photometry of Sex A and Sex B in electronic form. We thank the VLA-ANGST project for making their HI datacubes and maps publicly available. We also thank Jürgen Ott for helpful information on the data reduction. We are grateful to the anonymous referee for useful comments and suggestions that improved the overall quality and focus of the paper. This research has made use of the SIMBAD database, operated at the CDS, Strasbourg, France. This research has made use of the NASA/IPAC Extragalactic Database (NED) which is operated by the Jet Propulsion Laboratory, California Institute of Technology, under contract with the National Aeronautics and Space Administration. This research make use of SDSS data. Funding for the SDSS and SDSS-II has been provided by the Alfred P. Sloan Foundation, the Participating Institutions, the National Science Foundation, the US Department of Energy, the National Aeronautics and Space Administration, the Japanese Monbukagakusho, the Max Planck Society, and the Higher Education Funding Council for England. The SDSS Web Site is http: //www. sdss. org. The SDSS is managed by the Astrophysical Research Consortium for the Participating

12 See Bellazzini et al. (2013), for alternative hypotheses. 
Institutions. The Participating Institutions are the American Museum of Natural History, Astrophysical Institute Potsdam, University of Basel, University of Cambridge, Case Western Reserve University, University of Chicago, Drexel University, Fermilab, the Institute for Advanced Study, the Japan Participation Group, Johns Hopkins University, the Joint Institute for Nuclear Astrophysics, the Kavli Institute for Particle Astrophysics and Cosmology, the Korean Scientist Group, the Chinese Academy of Sciences (LAMOST), Los Alamos National Laboratory, the Max-Planck-Institute for Astronomy (MPIA), the Max-Planck-Institute for Astrophysics (MPA), New Mexico State University, Ohio State University, University of Pittsburgh, University of Portsmouth, Princeton University, the United States Naval Observatory, and the University of Washington.

\section{References}

Ahn, C. P., Alexandroff, R., Allende Prieto, C., et al. 2012, ApJS, 203, 21 Barkana, R., \& Loeb, A. 2001, Phys. Rep., 349, 125 Barnes, D. G., \& de Blok, W. J. G. 2001, AJ, 122, 825

Battaglia, G., Fraternali, F., Oosterloo, T., \& Sancisi, R. 2006, A\&A, 447, 49 Beccari, G., Pasquato, M., de Marchi, G., et al. 2010, ApJ, 713, 194 Bellazzini, M. 2004, MNRAS, 347, 119

Bellazzini, M. 2008. Mem. Soc. Astron. It., 79, 440 (B08)

Bellazzini, M., Ferraro, F. R., \& Pancino, E. 2001, ApJ, 556, 635

Bellazzini, M., Fusi Pecci, F., Messineo, M., Monaco, L., \& Rood, R. T. 2002a, AJ, 123, 1509

Bellazzini M., Ferraro F. R., Origlia L., et al. 2002b, AJ, 124, 3222

Bellazzini, M., Ferraro, F. R., Sollima, A., Pancino, E., \& Origlia, L. 2004, A\&A, 424, 199

Bellazzini, M., Beccari, G., Oosterloo, T. A., et al. 2011a, A\&A, 527, A58

Bellazzini, M., Perina, S., Galleti, S., \& Oosterloo, T. A. 2011b, A\&A, 533, A37

Bellazzini, M., Oosterloo, T., Fraternali. F., \& Beccari, G. 2013, A\&A, 559, L11

Bertin, E., \& Arnouts, S. 1996, A\&AS, 117, 393

Ciotti, L., \& Bertin, G. 1999, A\&A, 352, 447

Clem, J. L., Vandenberg, D. A., \& Stetson, P. B. 2008, AJ, 135, 628

Carretta, E., \& Gratton, R. 1997, A\&AS, 121, 95

Carretta, E., Bragaglia, A., Gratton, R. G., D’Orazi, V., \& Lucatello, S. 2009, A\&A, 508, 695

Corbelli, E., \& Salpeter, E. E. 1993, ApJ, 419, 104

Cotton, W. D., Condon, J. J., \& Arbizzani, E. 1999, ApJS, 125, 409

Dalcanton, J. J., Williams, B. F., Seth, A. C., et al. 2009, ApJS, 183, 67

Dekel, A., Birnboim, Y., Engel, G., et al. 2009, Nature, 457, 451

Dohm-Palmer, R. C., Skillman, E. D., Saha, A., et al. 1997a, AJ, 114, 2514

Dohm-Palmer, R. C., Skillman, E. D., Saha, A., et al. 1997b, AJ, 114, 2527

Dolphin, A. E., Saha, A., Skillman, E. D., et al. 2003, AJ, 126, 187

Federici, L., Bellazzini, M., Galleti, S., et al. 2007, A\&A, 473, 429

Ferguson, A. M. N, Irwin, M., Chapman, S., et al. 2007, in Island Universes, Astrophys. Space Sci. Proc. (Springer), 239

Gallart, C. 2008, ASP Conf. Ser., 390, 278

Galleti, S., Bellazzini, M., Federici, L., Buzzoni, A., \& Fusi Pecci, F. 2007, A\&A, 471, 127

Georgiev, I. Y., Puzia, T. H., Goudfrooij, P., \& Hilker, M. 2010, MNRAS, 406, 1967

Giallongo, E., Ragazzoni, R., Grazian, A. 2008, A\&A, 482, 349

Giovanelli, R., Haynes, M. P., Adams, E. 2013, AJ, 146, 15

Girardi, L., Grebel, E. K., Odenkirchen, M., \& Chiosi, C. 2004, A\&A, 422, 205

Johnston, K. V., Sigurdsson, S., \& Hernquist, L. 1999, MNRAS, 302, 771

Harbeck, D., Grebel, E. K., Holtzman, J., et al. 2001, AJ, 122, 3092

Harris, W. E.,1996, AJ, 112, 1487

Hidalgo, S. L., Aparicio, A., \& Gallart, C. 2008, AJ, 136, 2332

Hunter, D. A., \& Elmegreen, B. G. 2006, ApJ, 162, 49 (HE06)

Huxor, A. P., Tanvir, N. R., Irwin, M. J., et al. 2005, MNRAS, 360, 1007
Huxor, A. P., Ferguson, A. M. N., Veljanoski, J., Mackey, A. D., \& Tanvir, N. R. 2013, MNRAS, 429, 1039

Hwang, N., Lee, M. G., Lee, J. C., et al. 2011, ApJ, 738, 58

Kallivayalil, N., van der Marel, R. P., Besla, G., Anderson, J., \& Alcock, C. 2013, ApJ, 764, 161

Kazantzidis, S., Lokas, E. L., Callegari, S., Mayer, L., \& Moustakas, L. A. 2011, ApJ, 726, 98

Kaufer, A., Venn, K. A., Tolstoy, E., Pinte, C., \& Kudritzki, R.-P. 2004, AJ, 127, 2723

Keenan, D. W. 1981a, A\&A, 71, 245

Keenan, D. W. 1981b, A\&A, 95, 340

Kirby, E. N., Cohen, J. G., \& Bellazzini, M. 2012, ApJ, 751, 46

Kirby, E. N., Cohen, J. G., \& Bellazzini, M. 2013, ApJ, 768, 96

Klimentowski, J., Lokas, E. L., Kazantzidis, S., et al. 2009, MNRAS, 400, 2162

Kniazev, A. Y., Grebel, E. K., Pustilnik, S. A., Pramskij, A. G., \& Zucker, D. B. 2005, AJ, 130, 1558

Lauer, T. R. 1985, ApJS, 57, 473

Lovell, M. R., Eke, R. E., Frenk, C. S., \& Jenkins, A. 2011, MNRAS, 413, 3013

Magrini, L., Leisy, P., Corradi, R. L. M., et al. 2005, A\&A, 443, 115

Maloney, P. 1992, ApJ, 398, L89

Massey, P., Olsen, K. A. G., Hodge, P. W., et al. 2007, AJ, 133, 2393

Mateo, M. 1998, ARA\&A, 36, 435

Mayer, L., Kazantzidis, S., Mastropietro, C., \& Wadsley, J. 2007, Nature, 445, 738

McConnachie, A. 2012, AJ, 144, 4 (M12)

Monelli, M., Hidalgo, S. L., Stetson, P. B., et al. 2010a, ApJ, 720, 1225

Monelli, M., Gallart, C., Hidalgo, S. L., et al. 2010b, ApJ, 722, 1864

Muñoz, R. R., Majewski, S. R., \& Johnston, K. V. 2008, ApJ, 679, 346

Ott, J., Stilp, A. M., Warren, S. R., et al. 2012, AJ, 144, 123

Peacock, M. A., Zepf, S. E., Maccarone, T. J., \& Kundu, A. 2011, ApJ, 737, 5

Pedreros, M. H., \& Gallart, C. 2002, Rev. Mex. Astron. Astrofis., 14, 73

Peñarrubia, J., Navarro, J. F., \& McConnachie, A. W. 2008, ApJ, 673, 226

Peñarrubia, J., Navarro, J. F., McConnachie, A. W., \& Martin, N. F. 2009, ApJ, 698, 222

Penny, S. J., Pimbblet, K. A., Conselice, C. J., et al. 2012, ApJ, 758, L32

Pohlen, M., \& Trujillo, I. 2006, A\&A, 454, 759

Rockosi, C. M., Odenkirchen, M., \& Grebel, E. K. 2002, AJ, 124, 349

Sakai, S., Madore, B. F., \& Freedman, W. L. 1996, ApJ, 461, 713

Sanna, N., Bono, G., Stetson, P. B., et al. 2010, ApJ, 722, L244

Sawala, T., Scannapieco, C., Maio, U., \& White, S. 2010, MNRAS, 402, 1599

Sawala, T., Scannapieco, C., \& White, S. 2012, MNRAS, 420, 1714

Schlafly, E., \& Finkbeiner, D. P. 2011, ApJ, 737, 103

Schlegel, D. J., Finkbeiner, D. P., \& Davis, M. 1998, ApJ, 500, 525

Sérsic, J. L. 1968, Atlas de Galaxias Australes (Cordoba, Argent.: Obs. Astron.)

Sharina, M. E., Puzia, T. H., \& Krylatyh, A. S. 2007, Astroph. Bull., 62, 209

Sharina, M. E., Karachentsev, I. D., Dolphin, A. E., et al. 2008, MNRAS, 384, 1544

Shaya, E. J., \& Tully, R. B. 2013, MNRAS, 436, 2096

Shen, S., Madau, P., Conroy, C., Governato, F., \& Mayer, L. 2014, ApJ, submitted [arXiv: 1308.4131$]$

Stetson, P. B. 1987, PASP, 99, 191

Stetson, P. B. 1994, PASP, 106, 250

Teyssier, M., Johnston, K. V., \& Kuhlen, M. 2012, MNRAS, 426, 1808

Teyssier, R., Pontzen, A., Dubois, Y., \& Read, J. I. 2013, MNRAS, 429, 3068

Tolstoy, E., Hill, V., \& Tosi, M. 2009, ARA\&A, 47, 371

Tucker, D. L., Kent, S., Richmond, M. W., et al. 2006, Astron. Nachr., 327, 821

Tully, R. B., Rizzi, L., Dolphin, A. E., et al. 2006, AJ, 132, 729 (T06)

van den Bergh, S. 1999, ApJ, 517, L97

van der Kruit, P. C. 1979, A\&AS, 38, 15

Vansevičius, V., Arimoto, N., Hasegawa, T., et al. 2004, ApJ, 611, L93

Weisz, D. R., Dalcanton, J. J., Williams, B. F., et al. 2011, ApJ, 739, 5 
M. Bellazzini et al.: The extended structure of the dwarf irregular galaxies Sextans A and Sextans B
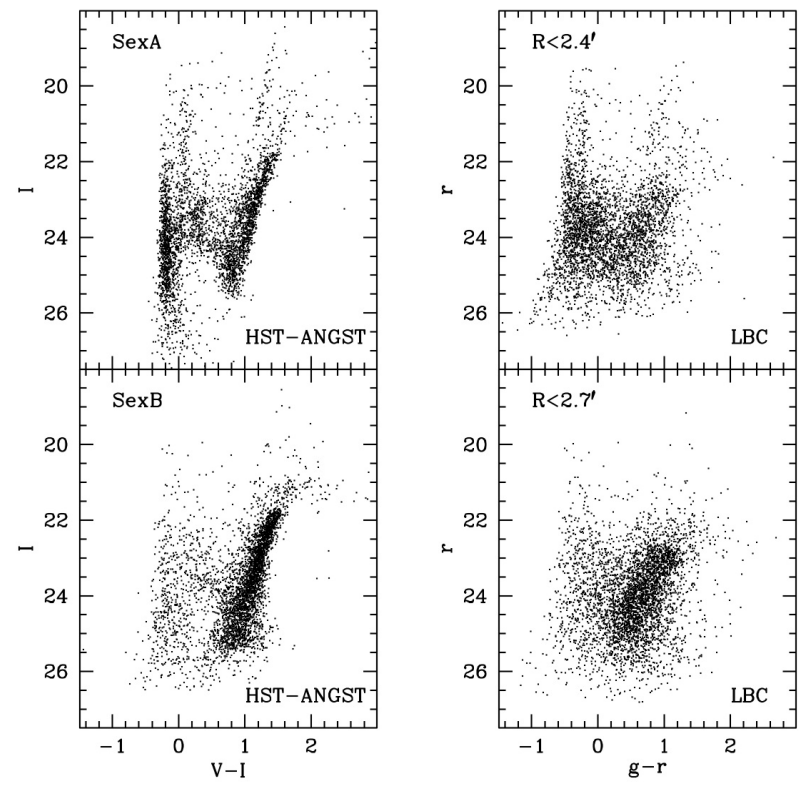

Fig. A.1. Comparison between HST photometry (left panels, from Dalcanton et al. 2009) and LBC photometry (right panels) for the stars in common between the two datasets.

\section{Appendix A: Comparison with HST photometry}

Colour magnitude diagrams from HST photometry provide the quality benchmark for crowded stellar systems like the distant (but resolved) galaxies we are considering within this project. Since deep HST photometry for Sex A and Sex B is publicly available from the ANGST database (Dalcanton et al. 2009), a direct comparison is worth performing.

In Fig. A.1 we show the CMDs obtained from the two datasets for the stars in common. Clearly our ground-based photometry cannot rival the top-level quality achievable from space, especially in the highly crowded central regions sampled by the HST data. Still, it is remarkable that all the features that can be identified in the LBC CMDs (e.g. the young MS and the parallel sequence of Blue Loop stars, the RSG plume) have a clear counterpart in the HST CMDs, thus indicating that the precision of our photometry is sufficient to reliably discriminate stars in different evolutionary phases.

\section{Appendix B: Compact star clusters}

It has been observed that dIrr galaxies of luminosity similar to Sex A and Sex B may host sizable globular clusters systems, with up to $\sim 10$ members (Georgiev et al. 2010). These clusters can provide very interesting insights into the early formation history of their parent galaxies (see, e.g. Hwang et al. 2011; Huxor et al. 2013, and references therein). The only reference to a systematic search for star clusters in Sex A and Sex B available in the literature is by Pedreros \& Gallart (2002); this was a brief contribution to the proceedings of a meeting and no candidate list was provided. On the other hand, Sharina et al. (2007) identified a relatively bright $\left(M_{V}=-7.8\right)$ and compact $\left(r_{\mathrm{h}} \simeq 4 \mathrm{pc}\right)$

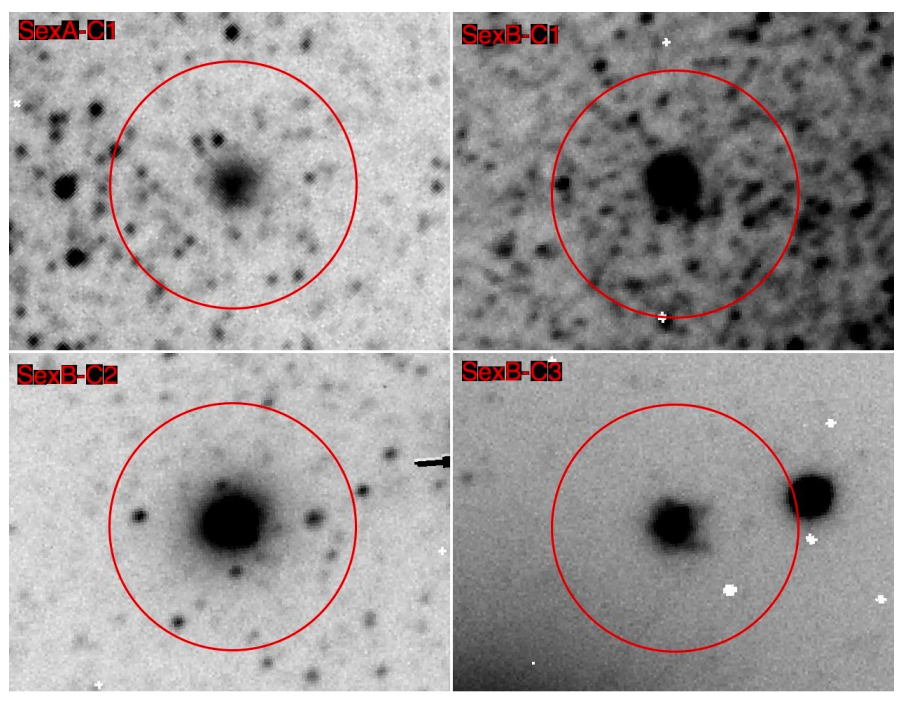

Fig. B.1. Stamp images of the cluster and candidate clusters listed in Table B.1. The circles around the objects have a radius of 10 arcsec; north is up, east to the left.

cluster in Sex B on HST images where it is marginally resolved into stars (Sex B-C1, hereafter). A low-resolution spectrum provided further confirmation of the membership of the cluster to Sex B as well as an estimate of the age ( $\simeq 2$ Gyr) and metallicity $([Z / H]=-1.35 \pm 0.3$, Sharina et al. 2007).

In the present contribution we focus our attention on compact clusters, i.e, objects that can resemble globular clusters, and old extended clusters as defined by Huxor et al. (2005), neglecting young associations, whose brightest stars are resolved in our images. We searched our image for compact star clusters by visual inspection and looking for sources more extended than the PSF in source catalogs obtained with Sextractor, as described in Galleti et al. (2007). Our search was not intended to be exhaustive, but to single out the best candidates. The images of the four objects we finally identified as the most reliable candidates are shown in Fig. B.1; their main properties are listed in Table B.1.

We re-identified Sex B-C1 and we found two additional candidates in Sex B and one in Sex A. We got the positions, integrated magnitudes, and measured FWHM from aperture photometry that we performed with Sextractor on the $g, r$ pair of deep images with the best seeing. All the candidates are clearly non-stellar objects, i.e, their light profile is significantly more extended than the PSF. It is reassuring that our estimate of the integrated $V$ magnitude for Sex B-C1 is in excellent agreement with Sharina et al. (2007), that report $V_{0}=17.90 \pm 0.02$. The case of Sex B-C1 shows that compact clusters cannot be unequivocally identified in our images (Fig. B.1). None of the presented candidates is particularly convincing. The hint of a diffuse halo and the relatively red colour suggest that Sex B-C2 may in fact be a roundish elliptical galaxy in the background (see Peacock et al. 2011). The fuzzy appearance of Sex B-C3 and Sex A-C1 is more akin to extended clusters (Huxor et al. 2005, 2013) than to classical globulars. Spectroscopic and/or high-spatial-resolution imaging follow up is clearly needed to establish the actual nature of these objects. 
A\&A 566, A44 (2014)

Table B.1. Clusters and candidate clusters.

\begin{tabular}{lcccccccr}
\hline \hline Name & $\alpha$ & $\delta$ & $g$ & $r$ & $V$ & $F W H M_{r}$ & $F W H M_{r} /\left\langle F W H M_{r, \text { stars }}\right\rangle$ & notes \\
\hline SexA-C1 & $10: 10: 51.7$ & $-04: 41: 33.5$ & 20.15 & 19.69 & 19.87 & $2.92^{\prime \prime}$ & 3.9 & cand. - extended? \\
\hline SexB-C1 & $10: 00: 04.7$ & $+05: 20: 07.5$ & 18.16 & 17.87 & 17.98 & $1.04^{\prime \prime}$ & 1.3 & Sharina et al. (2007) cluster \\
SexB-C2 & $10: 00: 07.3$ & $+05: 18: 00.8$ & 18.37 & 17.32 & 17.75 & $1.19^{\prime \prime}$ & 1.5 & cand. - E0 galaxy? \\
SexB-C3 & $09: 59: 39.7$ & $+05: 16: 36.0$ & 19.65 & 19.55 & 19.59 & $2.77^{\prime \prime}$ & 3.1 & cand. - extended? \\
\hline
\end{tabular}

Notes. Integrated magnitudes are computed within apertures of radius $=15 \mathrm{px}$ (corresponding to $3.4^{\prime \prime}$ ); typical uncertainties are $\simeq 0.05$ mag. 